\title{
A visco-elasto-plastic model for granular materials under simple shear conditions
}

\author{
I. Redaelli*1 ${ }^{*}$ C. di Prisco ${ }^{1}$ and D. Vescovi ${ }^{1}$ \\ ${ }^{1}$ Department of Civil and Environmental Engineering (DICA), Politecnico di Milano, Milan, Italy
}

\section{SUMMARY}

The numerical simulation of rapid landslides is quite complex mainly because constitutive models capable of simulating the mechanical behaviour of granular materials in the pre- and post-collapse regimes are still missing. The goal of this paper is to introduce a constitutive model capable of capturing the response of dry granular flows from quasi-static to dynamic conditions, in particular when the material experiences a sort of from solid to fluid phase transition. An ideal assembly of identical spheres under simple shear conditions is condsidered. In the constitutive model, void ratio and granular temperature have been chosen as state variables, and both shear and normal stresses are computed as the sum of two contributions: the quasi static and the collisional one. The former one is determined by using a perfect elasto-plastic model including the critical state concept, while the latter one is derived from the kinetic theory of granular gases. The evolution of the granular temperature, fundamentally governing the material phase transition, is obtained by imposing the kinetic fluctuating energy balance. The constitutive relationship has been integrated, under both constant pressure and constant volume conditions, and the influence of shear strain rate, initial void ratio and normal pressure on the mechanical response has been investigated. Copyright (c) 0000 John Wiley \& Sons, Ltd.

Received ...

KEY WORDS: constitutive modelling; high strain rates; granular flows; kinetic theories; critical state concept; simple shear conditions

\section{INTRODUCTION}

As is well known, rapid landslides are particularly dangerous for their catastrophic consequences both on the territory and in terms of human lives. For this reason, in the last decades, several

\footnotetext{
*Correspondence to: I. Redaelli, Department of Civil and Environmental Engineering (DICA), Politecnico di Milano, 20133 Milan, Italy. E-mail: irene.redaelli@ polimi.it

Copyright $(\mathrm{C} 0000$ John Wiley \& Sons, Ltd.
}

Prepared using nagauth.cls [Version: 2010/05/13 v2.00] 
numerical approaches have been developed $[1,2,3,4,5]$ with the aim of assessing the vulnerability and the risk for the environment and, eventually, designing mitigation measures. However, the theoretical analysis of the movement of granular masses is not straightforward since the considerable complexity of the phenomenon is still far to be understood. In order to describe both the inception and the propagation phase of the landslide, the numerical approach has to be capable of dealing with large displacements, large strain rates, heterogeneous and multiphase granular materials characterized by a particularly complex rheology, governed this latter by hydro-thermo-mechanical processes. During rapid landslides, because of the local high strain rates, a "from slide to flow" failure mechanism transition is also expected: when the velocity of the motion is sufficiently large, landslides may evolve into a fluid-like process where a large part of the granular material flows. For this reason, it is quite common in literature approaching the inception and the initiation by means of standard soil mechanics tools, whereas investigating the propagation by means of fluid dynamics. Some of the approaches available in the literature either treat the moving mass as a single phase incompressible fluid $[6,4,7]$, or artificially separate the initiation and the propagation stages without reproducing the transition from one to another [2,5].

To analyze both inception and propagation by using a unique numerical tool, according to the authors, a suitable constitutive model must be implemented, capable of capturing the macroscopic behavior of the material without disregarding its particulate nature and taking into consideration the complex microscale particle interaction mechanisms. In fact, as already suggested by some authors in the last decade $[8,9,10]$, at the micro-scale level, two are the possible dissipative mechanisms of interaction among particles: (i) enduring contacts among grains involved in force chains, and (ii) nearly instantaneous collisions. When the latter mechanism prevails, that is when the particles are widely spaced (i.e. the void ratio is sufficiently large), the material starts behaving like a viscous fluid and the stress tensor becomes prevalently dependent on the strain rate. Under this condition, usually called "collisional" regime, deformations are rapid and the material is strongly agitated: the energy of the system is totally dissipated through collisions and stored as kinetic energy due to the particle velocity fluctuations. Conversely, in the "quasi-static" regime (when force chains dominate the material response), strain rates are small and the entire network of contacts has to be continuously re-arranged. The energy is mainly stored as elastic energy and dissipated through frictional enduring contacts. When the grains interact both through force chains and through collisions, the material is in the "transition" regime. Understanding and reproducing the "phase transition" between the solid and the fluidized states is the most ambitious goal of this paper. The collisional regime has been largely 
studied in the context of kinetic theories of granular gases [11, 12, 13, 14, 15, 16]. In this context, the state variable representing the measure of the degree of agitation of the system is the granular temperature. In standard kinetic theories the granular material is assumed to be composed of identical, frictionless spheres which interact mainly through instantaneous, binary and uncorrelated collisions, but several modifications to standard kinetic theories have been recently introduced in the literature with the aim of taking into account interparticle friction $[17,18,14,19,20]$, correlated motion of particles [21, 22], nonspherical grains [23] and polydispersivity [24]. Nevertheless, kinetic theories are not yet capable of capturing the mechanical behavior of granular materials observed at high concentrations, when force chains develop within the medium [25, 26]. On the other hand, the constitutive models proposed within the soil mechanics community [27, 28, 29], considering quasistatic regimes and not incorporating the granular temperature as a representative state variable, are unable to reproduce the previously mentioned "from solid to fluid" state transition.

To achieve this goal, several constitutive models have been proposed in the literature, as those based on either dimensional analyses [30, 31, 32] or granular solid hydrodynamics [33]. In contrast, the theoretical constitutive model hereafter presented, as in Johnson and Jackson [34, 35], Savage [36], Louge [37], Lee and Huang [38], Berzi et al. [39] and Vescovi et al. [40], assumes a parallel scheme according to which the stress tensor is evaluated as the sum of two contributions: one "ratedependent" and another "rate-independent". The model hereafter illustrated can be interpreted as the extension to unsteady conditions of the model discussed in Berzi et al. [39] and Vescovi et al. [40]. The parallel scheme was already proposed by some authors [34, 35, 36, 37, 38], but the novelty of the approach introduced by Berzi et al. [39] and Vescovi et al. [40] concerns the role of critical state [41, 42, 43, 44, 28]: this is here interpreted as the limit for granular stationary flows, under simple shear conditions, when the granular temperature nullifies.

In Berzi et al. [39] and Vescovi et al. [40], as it is here in the following, a revised kinetic theory is adopted: this allows to account, in the collisional contribution, for both the clusters formation occuring at high concentrations [21,22] and the contact duration during a collision. In this paper the quasi-static contribution is computed by assuming a perfect elasto-plastic constitutive relationship including the critical state concept.

The paper is organized as follows. In Section 2, the constitutive equations are defined. In Section 3 , an introductive discussion of the theoretical model is presented. In Section 4, the constitutive equations are integrated in time, by applying suitable initial conditions. Constant pressure and constant volume tests are taken into account in order to study both the steady and unsteady responses 
under a wide range of both strain rate values and initial conditions. Finally, concluding remarks are in Section 5.

\section{THEORY}

As in Vescovi et al. [40], in this paper, an ideal assembly of identical spherical particles of diameter $d$ and density $\rho_{p}$ is taken into consideration. Simple shear conditions are imposed (Figure 1) and thus the components of the stress vector $\sigma$ governing the response of the material are the shear stress $\tau$ and the normal stress $\sigma$, whereas the components of the strain vector $\epsilon$ are the shear strain $\gamma$ and the normal strain $v$. The state variables taken into account are the void ratio $e$ (or, alternatively, the material concentration $\nu=1 /(1+e))$, and the granular temperature $T$, defined as

$$
T=\frac{\langle\langle\mathbf{V}\rangle\rangle}{3},
$$

where $\langle\langle\cdot\rangle\rangle$ designates the average over particle velocity using the single particle velocity distribution function, and $\mathbf{V}$ is the particle velocity fluctuation:

$$
\mathbf{V}=\mathbf{v}-\mathbf{u},
$$

being $\mathbf{v}$ the instantaneous single particle velocity and $\mathbf{u}=\langle\langle\mathbf{v}\rangle\rangle$ the mean velocity.

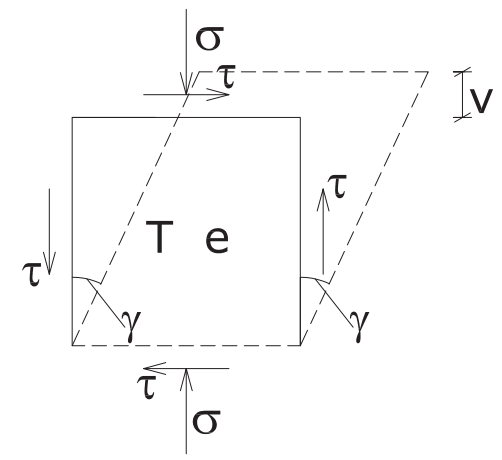

Figure 1. Representative elementary volume under simple shear conditions.

To evaluate the stress vector, a parallel scheme, as in Berzi et al. [39] and Vescovi et al. [40], is proposed (Figure 2). The quasi-static contribution, composed of an elastic spring acting in series with a frictional slider, is added to the collisional contribution represented by the damper.

As was already proposed by Johnson and Jackson [34, 35], Savage [36], Louge [37], Lee and Huang [38], Berzi et al. [39] and Vescovi et al. [40], the quasi-static contribution, $\boldsymbol{\sigma}_{q}$, is assumed to 
be associated with long elapsing frictional contacts among grains involved in force chains, whereas the collisional one, $\sigma_{c}$, with instantaneous inelastic collisions:

$$
\boldsymbol{\sigma}=\left[\begin{array}{l}
\sigma \\
\tau
\end{array}\right]=\boldsymbol{\sigma}_{q}+\boldsymbol{\sigma}_{c}=\left[\begin{array}{c}
\sigma_{q} \\
\tau_{q}
\end{array}\right]+\left[\begin{array}{l}
\sigma_{c} \\
\tau_{c}
\end{array}\right] .
$$

From a kinematic viewpoint, the model schematically reported in Figure 2 also implies

$$
\boldsymbol{\epsilon}=\left[\begin{array}{l}
v \\
\gamma
\end{array}\right]=\left[\begin{array}{l}
v_{q} \\
\gamma_{q}
\end{array}\right]=\left[\begin{array}{l}
v_{c} \\
\gamma_{c}
\end{array}\right]
$$

Subscripts $q$ and $c$ denote the quasi-static and the collisional contribution, respectively. According to the standard elasto-plastic theories,

$$
\epsilon=\epsilon^{e}+\epsilon^{p}
$$

where $\epsilon^{e}$ and $\epsilon^{p}$ are the elastic and the plastic components of $\epsilon$, respectively.

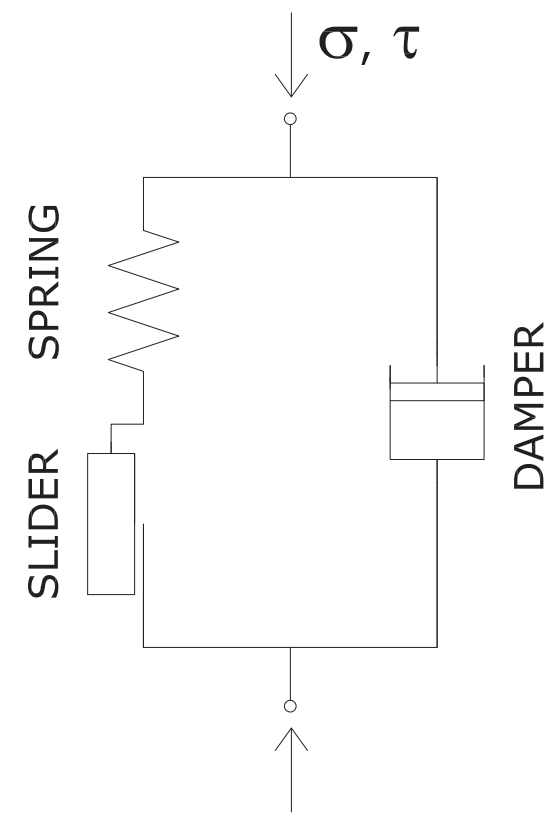

Figure 2. Rheological model for the granular material flowing under transient conditions.

For the system of Figure 2, treated as a material point, the total balance of energy reduces to:

$$
\dot{W}=\dot{E}_{k, f}+\dot{E}_{e l}+\Gamma,
$$


where dots stand for time derivative. In Equation 6, the energy produced by the work of internal stresses, $\dot{W}$, is defined as

$$
\dot{W}=\sigma \cdot \dot{\epsilon}
$$

$\dot{E}_{k, f}$, the kinetic fluctuating transported energy associated with the agitation of the material, is given, according to kinetic theories [15], by

$$
\dot{E}_{k, f}=\frac{3}{2} \rho_{p} \frac{1}{1+e} \dot{T}
$$

$\dot{E}_{e l}$, the variation in elastically stored energy, reads:

$$
\dot{E}_{e l}=\sigma_{q} \cdot \dot{\boldsymbol{\epsilon}}^{e}
$$

Finally, $\Gamma$ is the dissipated energy, that, according to the parallel scheme [40], can be written as

$$
\Gamma=\Gamma_{q}+\Gamma_{c}
$$

where $\Gamma_{q}$ is the energy dissipated by force chains

$$
\Gamma_{q}=\boldsymbol{\sigma}_{q} \cdot \boldsymbol{\epsilon}^{p}
$$

whereas $\Gamma_{c}$ is the energy dissipated by inelastic collisions. This term will be discussed in Section 2.2. Substituting Equations 8-11 into Equation 6 the following is obtained:

$$
\boldsymbol{\sigma}_{c} \cdot \dot{\boldsymbol{\epsilon}}=\frac{3}{2} \rho_{p} \frac{1}{1+e} \dot{T}+\Gamma_{c}
$$

that is the balance of the kinetic fluctuating energy.

\subsection{The quasi-static elasto-plastic constitutive contribution}

As is well known, elasto-plastic constitutive relationships have to be incrementally formulated:

$$
\dot{\boldsymbol{\sigma}}_{q}=\mathbf{D}^{e p} \dot{\boldsymbol{\epsilon}}
$$

where $\mathbf{D}^{\mathrm{ep}}$ is the elasto-plastic matrix $(2 \times 2)$. In the model hereafter introduced, three different mechanical responses are assumed: 
- the elastic response, for either $f<0$ or $f=0 \cap \dot{f}<0$;

- the standard perfect elasto-plastic response, for $f=\dot{f}=0$;

- the critical elasto-plastic response, for $f=\dot{f}=G_{1}=\dot{G}_{1}=0$.

where $f=0$ and $G_{1}=0$ are the yield and critical state loci, respectively. The expressions for $f$ and $G_{1}$, as well as the details about the conditions associated with the activation of the three different responses, are given here below.

\section{The elastic response}

In this case

$$
\mathbf{D}^{e p}=\mathbf{D}^{e},
$$

where $\mathbf{D}^{e}$ is the elastic stiffness matrix. This is derived by employing a hyperelastic formulation $[45,46,47,48,49,50,51]$. Its dependence on the stress state is imposed by suitably defining the Gibbs energy $E_{e l}$. The authors have decided to adopt, for defining this latter, the approach proposed by Houlsby et al. [51] and they have introduced, under simple shear conditions, the following expression:

$$
E_{e l}=\frac{p_{0}^{2-n}}{p_{a}^{1-n} \bar{k}(1-n)(2-n)}-\frac{\sigma_{q}}{\bar{k}(1-n)},
$$

where

$$
p_{0}^{2}=\sigma_{q}^{2}+\frac{\bar{k}(1-n) \tau_{q}^{2}}{\bar{g}}
$$

$p_{a}$ is the atmospheric pressure, $\bar{k}$ and $\bar{g}$ are material dimensionless constitutive parameters associated with the oedometric and the shear stiffnesses, respectively, whereas $n$ is a non-dimensional constant $(n \neq 1)$. From Equations 15 and 16 , the authors have derived the elastic matrix $\mathbf{D}^{e}$ as it follows:

$$
\mathbf{D}^{e}=\left[\frac{\partial^{2} E_{e l}}{\partial \boldsymbol{\sigma}_{q}^{2}}\right]^{-1}
$$

\section{The standard perfect elasto-plastic response}

In standard perfect elasto-plasticity [52, 53, 54], the elasto-plastic stiffness matrix is given by

$$
\mathbf{D}^{e p}=\mathbf{D}^{e}-\frac{\mathbf{D}^{e} \frac{\partial g}{\partial \boldsymbol{\sigma}_{q}}\left(\frac{\partial f}{\partial \boldsymbol{\sigma}_{q}}\right)^{T} \mathbf{D}^{e}}{\left(\frac{\partial f}{\partial \boldsymbol{\sigma}_{q}}\right)^{T} \mathbf{D}^{e} \frac{\partial g}{\partial \boldsymbol{\sigma}_{q}}},
$$

where $g$ is the plastic potential. Here below, the expressions for both $f$ and $g$ are given.

Yield locus 
The yield locus $f=0$ governs the activation of the frictional slider (Figure 2), and its expression is given by:

$$
f= \begin{cases}\beta\left(\frac{\left|\tau_{q}\right|}{\sigma_{q}^{*}}\right)^{3 / 2} \sigma_{q}^{*}-\sin \left(\frac{\sigma_{q}}{\sigma_{q}^{*}}\right) \sigma_{q}^{*}=0 & \sigma_{q}<\sigma_{q 0} \\ \left|\tau_{q}\right|-\bar{\tau}_{q}-\sigma_{q} \tan \phi^{\prime}=0 & \sigma_{q} \geq \sigma_{q 0}\end{cases}
$$

where

$$
\begin{aligned}
\sigma_{q}^{*} & =\frac{\sigma_{q 0}}{\arccos \left(\tan \phi^{\prime}\right)} \\
\beta & =\left(\frac{2}{3} \frac{1}{\left[\sin \left(\arccos \left(\tan \phi^{\prime}\right)\right)\right]^{1 / 3}}\right)^{3 / 2} \\
\bar{\tau}_{q} & =\frac{3}{2} \sigma_{q 0} \frac{\sin \left(\arccos \left(\tan \phi^{\prime}\right)\right)}{\arccos \left(\tan \phi^{\prime}\right)}-\sigma_{q 0} \tan \phi^{\prime} .
\end{aligned}
$$

$\phi^{\prime}$ is the internal friction angle at the critical state under simple shear conditions [43, 54, 55], whereas $\sigma_{q 0}$ is a constant non influencing the mechanical response of the model and whose geometrical meaning is graphycally suggested in Figure 3. The expression of Equation 19 allows to avoid the angular point implicitely introduced by the Mohr-Coulomb locus and implies the differentiability of $f$ even in the origin. A comparison between the standard Mohr-Coulomb locus and the locus defined in Equation 19 is reported in Figure 3.

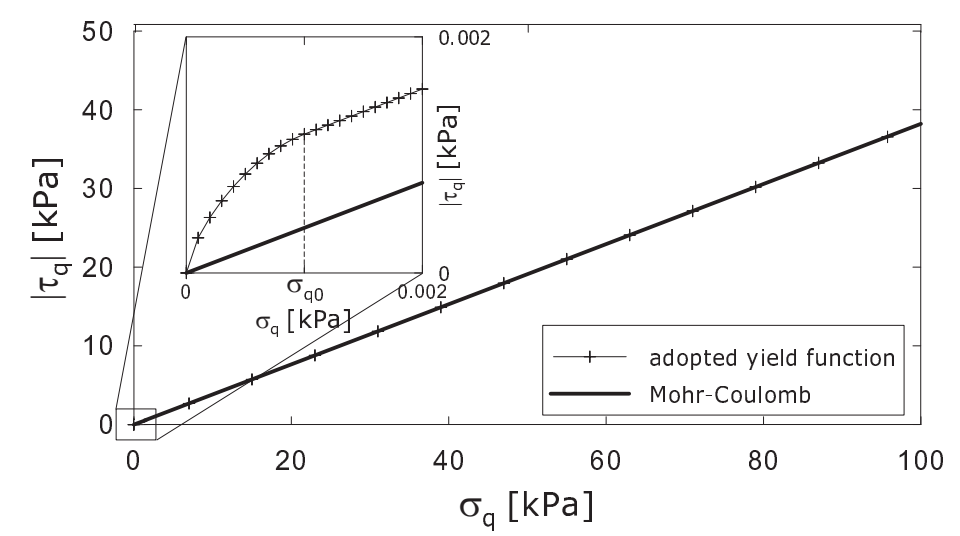

Figure 3. Comparison between the expression for $f=0$ here proposed (Equation 19) and the standard MohrCoulomb.

\section{Plastic potential}

With the aim of incorporating the critical state concept into the model, a plastic potential evolving with the void ratio is here introduced:

$$
g=\left|\tau_{q}\right|+\delta G_{1}
$$


where $\delta$ is a positive parameter and

$$
G_{1}=\frac{\sigma_{q}}{K}-f_{0}
$$

The critical state locus in the $e-\sigma_{q}$ plane is

$$
G_{1}=0
$$

as defined in Vescovi et al. [40]. In Equation 22,

$$
f_{0}= \begin{cases}a \frac{e-e_{c}}{(1+e)\left(1+e_{c}\right)} & e<e_{c} \\ 0 & e \geq e_{c} .\end{cases}
$$

and $K$ is the particle stiffness defined according to the Hertzian contact model $\left(K=4 E_{p} /[6(1-\right.$ $\left.\nu_{p}^{2}\right)$ ], being $E_{p}$ and $\nu_{p}$ the particle Young modulus and the Poisson coefficient, respectively). The expression for $f_{0}$ (Equation 24) coincides with that suggested by Chialvo et al. [56] fitting their 3D Discrete Element Method (DEM) numerical results concerning steady simple shear flows of monodisperse spherical particles. According to Chialvo et al. [56], $a$ is a dimensionless coefficient, related to the interparticle friction coefficient $\phi_{p}$ and $e_{c}$ is the critical void ratio describing the transition to the purely collisional regime under steady simple shear conditions (i.e. $\sigma_{q}=\tau_{q}=0$ ).

The current void ratio, implicitely appearing in Equation 21, is calculated by imposing the mass balance under uniform conditions:

$$
\frac{\dot{e}}{1+e}=-\dot{v}
$$

In standard perfect elasto-plasticity $[52,53,54]$, the plastic potential governs the direction of the plastic strain rate vector by means of the flow rule. From Equation 21, term $\partial g / \partial \boldsymbol{\sigma}_{q}$ of Equation 18 can be derived:

$$
\frac{\partial g}{\partial \boldsymbol{\sigma}_{q}}=\left[\begin{array}{c}
\frac{\partial g}{\partial \sigma_{q}} \\
\frac{\partial g}{\partial \tau_{q}}
\end{array}\right]=\left[\begin{array}{c}
\delta G_{1} \\
\operatorname{sgn}\left(\tau_{q}\right)
\end{array}\right] .
$$

From Equations 22, 24 and 26, it is evident that dilatancy $D=\dot{v}^{p} / \dot{\gamma}^{p}=\left(\partial g / \partial \sigma_{q}\right) /\left(\partial g / \partial \tau_{q}\right)$ is a function of both $e$ and $\sigma_{q}$ (state dependent dilatancy [44, 57]). In particular, when $G_{1}=0$, plastic volumetric strain rates nullify $(D=0)$, whereas when $G_{1}>0$ (loose conditions) the material experiences a plastic compaction $(D>0)$, and when $G_{1}<0$ (dense conditions) the material plastically dilates $(D<0)$. 


\section{The critical elasto-plastic response}

When the critical state condition is attained $\left(f=\dot{f}=G_{1}=0\right), D^{e p}$ is replaced by the critical elasto-plastic matrix $\mathbf{D}^{c r}$. This latter is obtained by imposing a sort of extended consistency rule:

$$
\dot{G}_{1}=0
$$

instead of imposing the standard flow rule. As a consequence

$$
\mathbf{D}^{c r}=\left[\begin{array}{cc}
\frac{\partial G_{1}}{\partial e}\left(\frac{\partial G_{1}}{\partial \sigma_{q}}\right)^{-1}(1+e) & 0 \\
\frac{\partial f}{\partial \sigma_{q}} \frac{\partial G_{1}}{\partial e}\left(\frac{\partial f}{\partial \tau_{q}} \frac{\partial G_{1}}{\partial \sigma_{q}}\right)^{-1}(1+e) & 0
\end{array}\right] .
$$

\subsection{The collisional contribution}

The collisional contribution is modelled according to kinetic theories of granular gases [11, 12, 13, 14, 15, 16]. According to these theories:

$$
\begin{aligned}
\sigma_{c} & =\left\langle\rho_{p} f_{1} T+\rho_{p} d f_{4} T^{1 / 2} \dot{v}\right\rangle \\
\tau_{c} & =\rho_{p} d f_{2} T^{1 / 2} \dot{\gamma} .
\end{aligned}
$$

where $\langle\cdot\rangle$ means $\langle X\rangle=0$ when $X<0$ and $\langle X\rangle=X$ when $X \geq 0$. In compact form, Equation 29 becomes

$$
\boldsymbol{\sigma}_{c}=\boldsymbol{\Phi}^{v} \dot{\boldsymbol{\epsilon}}+\mathbf{h},
$$

where $\boldsymbol{\Phi}^{v}$ is a viscous matrix, depending on $e$ and $T$, defined as:

$$
\boldsymbol{\Phi}^{v} \equiv\left\{\begin{array}{lc}
{\left[\begin{array}{cc}
\rho_{p} d f_{4} T^{1 / 2} & 0 \\
0 & \rho_{p} d f_{2} T^{1 / 2}
\end{array}\right]} & \sigma_{c}>0 \\
{\left[\begin{array}{cc}
0 & 0 \\
0 & \rho_{p} d f_{2} T^{1 / 2}
\end{array}\right]} & \sigma_{c}=0
\end{array}\right.
$$


whereas $\mathbf{h}$ is a vector depending on $e$ and $T$ according to the following expression:

$$
\mathbf{h} \equiv\left\{\begin{array}{cc}
{\left[\begin{array}{cc}
\rho_{p} f_{1} T \\
0
\end{array}\right]} & \sigma_{c}>0 \\
0 & \sigma_{c}=0 .
\end{array}\right.
$$

For evaluating the granular temperature $T$ and its evolution, Equation 12 is used. In Equation 12, the expression for $\Gamma_{c}$ is derived from the extended kinetic theory [21, 22]:

$$
\Gamma_{c}=\rho_{p} \frac{f_{3}}{L} T^{3 / 2}
$$

where the correlation length $L$ is a scalar variable representing the length of the contact chains:

$$
L=d \cdot \max \left(1, \frac{1}{2} c G^{1 / 3} \frac{d}{T^{1 / 2}}\left[\left(\dot{v}^{2}+\dot{\gamma}^{2}\right)^{1 / 2}+\dot{v}\right]\right)
$$

with $c$ a dimensionless material coefficient of order one.

In Equations 29, 33 and 34, $f_{1}, f_{2}$ and $G$ coincide with those proposed by Vescovi et al. [40] for the steady state case, whereas functions $f_{3}$ and $f_{4}$ are derived from Garzó and Dufty [15]. All these functions depend on $e$ and diverge for $e=e_{s}$, defined as the shear rigidity void ratio [58]. Their expression is in Table I. Functions $f_{i}, i=1, \cdots, 4$ depend also on the effective coefficient of restitution $\epsilon_{r}$, accounting for the energy dissipated during inelastic collisions and depending on the normal coefficient of restitution, the tangential coefficient of restitution in a sticking collision, and the interparticle friction coefficient, characterizing sliding collisions [20].

The current value of $T$, appearing in Equations 31-34, is obtained by integrating the balance of the kinetic fluctuating energy (Equation 12), in which Equation 33 is introduced:

$$
\dot{T}=\frac{\boldsymbol{\sigma}_{c} \cdot \dot{\boldsymbol{\epsilon}}-\rho_{p} \frac{f_{3}}{L} f_{4} T^{3 / 2}}{\frac{3}{2} \rho_{p} \frac{1}{1+e}}
$$

\subsection{The constitutive relationship}

According to Equation 3, in order to formulate the constitutive relationship, the quasi-static and the collisional contibutions defined in Sections 2.1 and 2.2, have to be added. To this purpose, the 
quasi-static contribution is expressed in finite form by integrating in time Equation 13:

$$
\sigma_{q}=\mathbf{D}^{e p} \Delta t \dot{\boldsymbol{\epsilon}}+\overline{\boldsymbol{\sigma}}_{q}
$$

where $\Delta t$ is the integration time step and $\bar{\sigma}_{q}$ is the quasi-static stress vector calculated at the previous time step. From Equations 30 and 36, the total stress vector becomes:

$$
\boldsymbol{\sigma}=\mathbf{D}^{v e p} \dot{\boldsymbol{\epsilon}}+\mathbf{c},
$$

Table I. List of expressions for the collisional contribution.

$$
\begin{aligned}
& f_{1}=4 \frac{G F}{1+e} \\
& f_{2}=\frac{8}{5 \pi^{1 / 2}} \frac{J G}{(1+e)} \\
& f_{3}=\frac{144}{5 \sqrt{2}} \frac{1}{(1+e)^{2}} \zeta^{*} \\
& f_{4}=\frac{5}{96} \pi^{1 / 2}\left[\frac{3}{5} \Gamma^{*}-\frac{2}{3} \eta_{k}^{*}\left(1+\frac{4}{5} \frac{g_{0}}{1+e}\left(1+\epsilon_{r}\right)\right)\right] \\
& G=\frac{g_{0}}{1+e} \\
& g_{0}= \begin{cases}\frac{(2 e+1)(1+e)^{2}}{2 e^{3}}, & \text { if } e \geq 1.04 \\
5.69\left(0.51-0.49 e_{s}\right) \frac{1+e}{e-e_{s}}, & \text { if } e<1.04\end{cases} \\
& F=\frac{1+\epsilon_{r}}{2}+\frac{1}{4 G} \\
& J=\frac{1+\epsilon_{r}}{2}+\frac{\pi}{32} \frac{\left[5+2\left(1+\epsilon_{r}\right)\left(3 \epsilon_{r}-1\right) G\right]\left[5+4\left(1+\epsilon_{r}\right) G\right]}{\left[24-6\left(1-\epsilon_{r}\right)^{2}-5\left(1-\epsilon_{r}^{2}\right)\right] G^{2}} \\
& \Gamma^{*}=\frac{128}{5 \pi} \frac{g_{0}}{1+e}\left(1+\epsilon_{r}\right)\left(1-\frac{1}{32} c^{*}\right) \\
& \eta_{k}^{*}=\left(g_{0}\left[1-\frac{1}{4}\left(1-\epsilon_{r}\right)^{2}\right]\left[1-\frac{1}{64} c^{*}\right]-\frac{5}{24} g_{0}\left(1-\epsilon_{r}^{2}\right)\left(1+\frac{3}{32} c^{*}\right)\right)^{-1}\left[1-\frac{2}{5}\left(1+\epsilon_{r}\right)\left(1-3 \epsilon_{r}\right) \frac{g_{0}}{1+e}\right] \\
& c^{*}=32\left(1-\epsilon_{r}\right)\left(1-2 \epsilon_{r}^{2}\right)\left[81-17 \epsilon_{r}+30 \epsilon_{r}^{2}\left(1-\epsilon_{r}\right)\right]^{-1} \\
& \zeta^{*}=\zeta^{0 *}+\zeta^{1 *} \\
& \zeta^{0 *}=\frac{5}{12} g_{0}\left(1+\epsilon_{r}^{2}\right)\left(1+\frac{3}{32} c^{*}\right) \\
& \zeta^{1 *}=\left[\frac{5}{96} \sqrt{\frac{\pi}{T}} d(1+e)\left(p^{*}-1\right)-\frac{5}{32}\left(1-\epsilon_{r}^{2}\right)\left(1+\frac{3}{64} c^{*}\right) g_{0} C_{d}\right] \dot{v} \\
& C_{d}=\frac{5}{96} \sqrt{\frac{5}{96}} d(1+e)\left[\frac{1}{2} \zeta^{0 *}+\nu_{\gamma}^{*}+\frac{5}{64} c^{*}\left(1+\frac{3}{64} c^{*}\right) g_{0}\left(1-\epsilon_{r}^{2}\right)\right]^{-1}\left[\frac{4}{15} \lambda \frac{g_{0}}{1+e}+\left(p^{*}-1\right)\left(\frac{2}{3}-\epsilon_{r}\right) c^{*}\right] \\
& p^{*}=1+2\left(1+\epsilon_{r}\right) \frac{g_{0}}{1+e} \\
& \nu_{\gamma}^{*}=\frac{1+\epsilon_{r}}{48} g_{0}\left[128-96 \epsilon_{r}+15 \epsilon_{r}^{2}-15 \epsilon_{r}^{3}+\frac{c^{*}}{64}\left(15 \epsilon_{r}^{3}-15 \epsilon_{r}^{2}+498 \epsilon_{r}-434\right)\right] \\
& \lambda=\frac{3}{8}\left[\left(1-\epsilon_{r}\right)\left(5 \epsilon_{r}^{2}+4 \epsilon_{r}-1\right)+\frac{c^{*}}{12}\left(159 \epsilon_{r}+3 \epsilon_{r}^{2}-19 \epsilon_{r}-15 \epsilon_{r}^{3}\right)\right] .
\end{aligned}
$$


where

$$
\mathbf{D}^{v e p} \equiv \mathbf{D}^{e p} \Delta t+\boldsymbol{\Phi}^{v}
$$

is the visco-elasto-plastic matrix, and

$$
\mathbf{c} \equiv \overline{\boldsymbol{\sigma}}_{q}+\mathbf{h}
$$

$\mathbf{D}^{\text {vep }}$ and $\mathbf{c}$ depend on the void ratio, the granular temperature $T$ and the quasi-static stress vector, evaluated at the previous time step.

\subsection{Constitutive parameters}

To define the constitutive relationship, 14 parameters have been introduced: 5 are micro-mechanical and refer to the single particle $\left(\rho_{p}, d, E_{p}, \nu_{p}\right.$ and $\left.\epsilon_{r}\right)$ and 9 are macro-mechanical $(\bar{k}, \bar{g}, n, \delta, c, a$, $\phi^{\prime}, e_{c}$ and $\left.e_{s}\right)$. For all the reference tests discussed in the following, the authors have employed the values listed in Table II. All the values assigned to the micro-mechanical parameters, except for the elastic constants $E_{p}$ and $\nu_{p}$, coincide with those chosen by Vescovi et al. [40]. As was previously mentioned, the authors, as far as the elastic contact rules among grains are concerned, decided to use, conversely to Vescovi et al. [40], a Hertzian relationship. As previously mentioned, the material is assumed to be composed of spherical grains $(d=0.001 \mathrm{~mm})$ made of quartz $\left(\rho_{p}=2600 \mathrm{~kg} / \mathrm{m}^{3}\right.$, $\left.E_{p}=90 \mathrm{GPa}, \nu_{p}=0.2\right)$.

Among the 9 macro-mechanical parametes, $\bar{k}, \bar{g}$ and $n$ are employed to define the Gibbs energy function (Equation 15), $\delta$ for the plastic potential (Equation 21), $a, e_{c}, \phi^{\prime}$ for the critical state locus (Equations 23 and 19) and $e_{s}$ and $c$ for the collisional functions (Table I). The values of $a, e_{c}$ and $\phi^{\prime}$ employed are taken from Chialvo et al. [56]. These have been calculated by performing 3D DEM numerical simulations on a granular assembly of identical spheres, for an interparticle friction coefficient $\phi_{p}=0.5$. The values of both $c$ and $\epsilon_{r}$ coincide with that used in Vescovi et al. [40]. In the definition of $f_{i}, i=1, \cdots, 4$ and $G$ (Table I), $e_{s}$ is a singular point. To avoid numerical problems, $e_{s}$ is imposed to be equal to 0.35 . The authors are aware of the arbitrariness of this choice but this is imposed by the type of functions $f_{i}$ here employed. A discussion about such a point is in Vescovi [59].

The unique constitutive parameters requiring, according to the authors, a suitable discussion, due to the lack of either experimental or numerical data, are $\delta, \bar{k}, \bar{g}$ and $n$. Nevertheless, for the sake of brevity, the results of the parametric study performed to assess the influence of $\delta, \bar{k}, \bar{g}$ and $n$ is 
not here reported. These parameters prevalently affect the volumetric behaviour and the rapidity in getting the steady state but not the nature of the mechanical response.

Table II. List of micro- and macro-mechanical parameters adopted in the tests.

\begin{tabular}{c}
\hline Micro-mechanical parameters \\
\hline$\rho_{p}=2600 \mathrm{~kg} / \mathrm{m}^{3}$ \\
$d=0.001 \mathrm{~m}$ \\
$E_{p}=90 \mathrm{GPa}$ \\
$\nu_{p}=0.2$ \\
$\epsilon_{r}=0.6$ \\
\hline Macro-mechanical parameters \\
$\bar{k}=381$ \\
$\bar{g}=127$ \\
$n=0.5$ \\
$\delta=0.1$ \\
$c=0.5$ \\
$a=0.089$ \\
$\phi^{\prime}=0.365 \mathrm{rad}$ \\
$e_{c}=0.70358$ \\
$e_{s}=0.35$
\end{tabular}

\section{THEORETICAL DISCUSSION}

As was previously sinthetically inferred, the model presented in this paper has been conceived to numerically reproduce the mechanical behaviour of a granular material under unsteady conditions. According to the model, the response of the material under unsteady conditions, can be: (I) viscoelastic, (II) visco-elasto-plastic, (III) critical and (IV) collisional, here interpreted as flow regimes. A graphical representation of the four regimes is in Figure 4. Each regime corresponds to a specific constitutive relationship which is derived from the general one given by Equation 37. The space of the possible evolving states in Figure 4 is bounded by two limit conditions: the static lower boundary, defined by $T=\dot{v}=\dot{\gamma}=0$ and the steady upper boundary, characterized by $\dot{T}=\dot{v}=\ddot{\gamma}=0$.

Here below, the constitutive relationship associated with each unsteady regime is discussed. 
(I) In the visco-elastic regime, conditions $f<0$ and $G_{1} \neq 0 \cap f=0, \dot{f} \neq 0$ and $G_{1} \neq 0$ are satisfied. The constitutive relationship simply reduces to

$$
\boldsymbol{\sigma}=\left(\mathbf{D}^{e} \Delta t+\boldsymbol{\Phi}^{v}\right) \dot{\boldsymbol{\epsilon}}+\mathbf{c},
$$

where $\boldsymbol{D}^{e}, \boldsymbol{\Phi}^{v}$ and $\mathbf{c}$ are given by Equations 17, 31 and 39, respectively. The material behaves like a visco-elastic solid and strain rates are sufficiently low. As a consequence, $T$ is almost negligible and the energy is predominantly stored as elastic energy.

(II) In the visco-elasto-plastic regime, conditions $f=\dot{f}=0$ and $G_{1} \neq 0$ are fulfilled and the constitutive relationship is

$$
\sigma=\mathbf{D}^{v e p} \dot{\boldsymbol{\epsilon}}+\mathbf{c}
$$

where $\mathbf{D}^{\text {vep }}$ is given by Equation 38. Quasi-static irreversible strains develop and their relevance with respect to the total irreversible strains is governed by $\dot{\gamma}$.

(III) In the critical regime, conditions $f=\dot{f}=G_{1}=\dot{G}_{1}=0$ and $e<e_{c}$ are satisfied. The constitutive relationship is defined as it follows:

$$
\boldsymbol{\sigma}=\left(\mathbf{D}^{c r} \Delta t+\mathbf{\Phi}^{v}\right) \dot{\boldsymbol{\epsilon}}+\mathbf{c},
$$

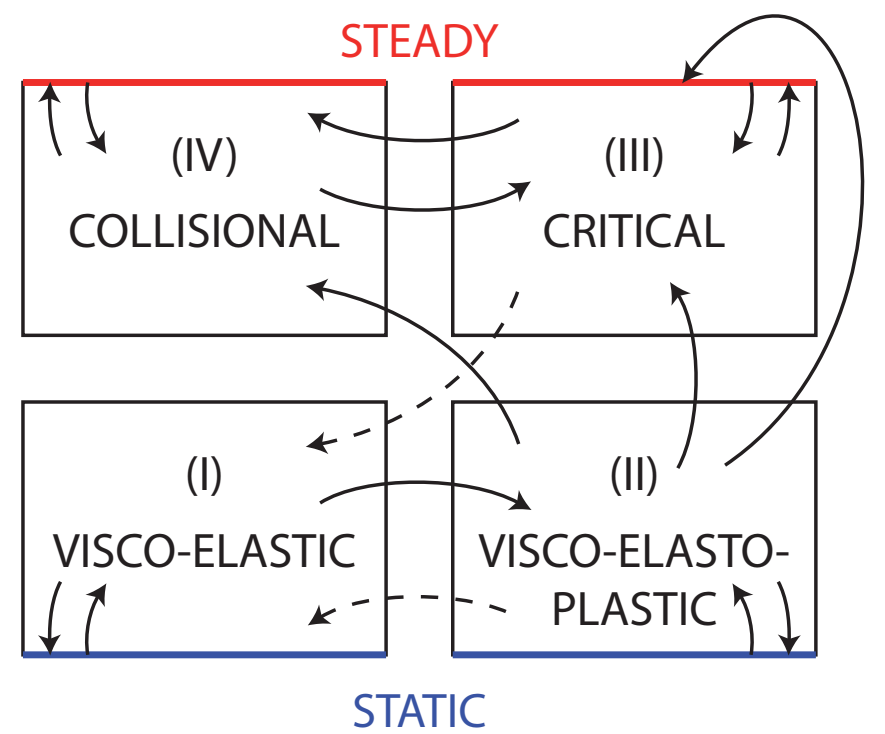

Figure 4. Schematical representation of the four unsteady flow regimes. The solid arrows and the dashed arrows corresponds to the possible loading $(\dot{\gamma}>0)$ or unloading $(\dot{\gamma}<0)$ paths, respectively. 
with $\mathbf{D}^{c r}$ given by Equation 28 and the response is visco-elasto-plastic. When steady conditions are reached (i.e. the state of the material lies on the upper boundary of regime (III)), conditions $\dot{T}=\dot{v}=\ddot{\gamma}=0$ are also satisfied and the work of the internal stresses is totally dissipated by both collisions and force chains. In this case, the balance of the kinetic fluctuating energy (Equation 35) provides

$$
T=d^{2} f_{5} \dot{\gamma}
$$

where

$$
f_{5}=\frac{L}{d} \frac{f_{2}}{f_{3}}
$$

and the correlation length, defined by Equation 34, reduces to

$$
L=d \cdot \max \left[1,\left(\frac{c^{2} G^{2 / 3} f_{3}}{4 f_{2}}\right)\right]
$$

As a consequence, Equation 42 becomes

$$
\begin{aligned}
& \sigma=K f_{0}+\rho_{p} d^{2} f_{1} f_{5} \dot{\gamma}^{2} \\
& |\tau|=K f_{0} \tan \phi^{\prime}+\rho_{p} d^{2} f_{2} f_{5}^{1 / 2} \dot{\gamma}^{2} .
\end{aligned}
$$

(IV) In the collisional regime, conditions $f=\dot{f}=G_{1}=\dot{G}_{1}=0$ and $e>e_{c}$ are satisfied. In this regime the material behaves like a rate-dependent collisional granular matter and the constitutive relationship becomes:

$$
\boldsymbol{\sigma}=\Phi^{v} \dot{\boldsymbol{\epsilon}}+\mathbf{h}
$$

where $\mathbf{h}$ is given by Equation 32. The response is purely collisional (i.e. force chains cannot develop and $\sigma_{q}=\tau_{q}=0$ ). The energy is solely stored as kinetic energy due to velocity fluctuations and is dissipated through collisions. The upper bound of this regime is again the steady state condition $(\dot{T}=\dot{v}=\ddot{\gamma}=0)$. When steady state is reached, the work of internal stresses is totally dissipated by collisions and the model reduces to:

$$
\begin{aligned}
& \sigma=\rho_{p} d^{2} f_{1} f_{5} \dot{\gamma}^{2} \\
& |\tau|=\rho_{p} d^{2} f_{2} f_{5}^{1 / 2} \dot{\gamma}^{2} .
\end{aligned}
$$


According to Figure 4, from regime (I) only regime (II) can be got. In contrast, from regime (II), according to the current $\dot{\gamma}$ and $e$ values, all the other regimes can be got. It is possible to go from (II) to (I) in case of unloading. From regime (IV) solely regime (III) can be got, whereas from regime (III) (during loading) regime (IV) and (during unloading) regime (I) can be achieved. For instance, standard quasi-static loading tests, starting from the lower boundary of regime (I) (static condition), passes through regime (II) and gets regime (III) on its upper bound (steady state).

\section{NUMERICAL RESULTS}

In this section, the model is discussed with reference to constant pressure and constant volume tests and by discussing the influence of both strain rate level and initial conditions. Both loading $(\dot{\gamma}>0)$ and unloading $(\dot{\gamma}<0)$ tests are accounted for.

\section{Initial conditions}

Here in the following, the authors have assumed that at time $t=t_{0}$ the material may be in either (i) static or (ii) stationary conditions. In any case, at time $t=t_{0}, \dot{\gamma}_{0}, \dot{v}_{0}, e_{0}, T_{0}, \tau_{0}, \sigma_{0}$ have to be either directly assigned or determined by imposing a constraint. The subscript 0 refers to quantities evaluated at time $t=t_{0}$.

\section{(i) Static conditions}

In this case, three constraints have to be imposed: $T_{0}=\dot{\gamma}_{0}=\dot{v}_{0}=0$ and three variables must be initially assigned $\left(e_{0}, \sigma_{0}\right.$ and $\left.\tau_{0}\right)$. Actually, according to Equation $12, T$ can never nullify. Therefore the $T_{0}$ value will be assigned very small but not nil. Nevertheless, it is possible to prove that, if a sufficiently small value of $T_{0}$ is imposed, an inifinitesimal variation of this initial datum does not affect the system response. Under static conditions, the initial collisional contribution is negligible (Equation 29) and this implies that $\sigma_{0} \approx \sigma_{q 0}$ and $\tau_{0} \approx \tau_{q 0}$.

$\sigma_{q 0}, \tau_{q 0}$ and $e_{0}$ are free variables but they cannot be arbitrarily assigned: in Figure 5 the admissible regions for these variables are plotted in the $\tau_{q}-\sigma_{q}$ and $e-\sigma_{q}$ planes. In particular, $f \leq 0$ condition has to be satisfied at $t=t_{0}$ and this implies that, in Figure 5a, the image point of the quasi-static state of stress has to belong to the grey domain. Moreover, in the $e-\sigma_{q}$ plane (Figure 5b), the initial state must belong to either the light or the dark grey sub-domains. These sub-domains are obtained by drawing three lines: the upper one, representing the maximum void ratio under static conditions, is taken from the literature [60]. This was numerically obtained by performing 3D DEM simulations on disordered packing of 
identical spheres:

$$
e=\frac{K b e_{\max }+\sigma_{q}\left(1+e_{\max }\right)}{K b-\sigma_{q}\left(1+e_{\max }\right)}
$$

where $b=0.545$ and $e_{\max }$ corresponds to the loosest possible packing of randomly distributed monodispersed spheres under static conditions for $\sigma_{q}=0$. According to Silbert [60], for $\phi_{p}=$ $0.5, e_{\max }=0.74216$. The intermediate line of Figure $5 \mathrm{~b}$ is the critical state line (Equation 23) while the lower one, corresponding to the densest possible packing of randomly distributed monodispersed spheres under static conditions, is totally theoretical and, for the sake of simplicity, it is here assumed to be horizontal with $e=e_{\min }=0.57$.

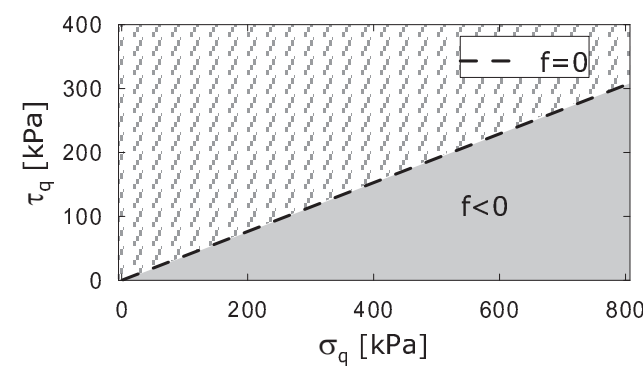

(a)

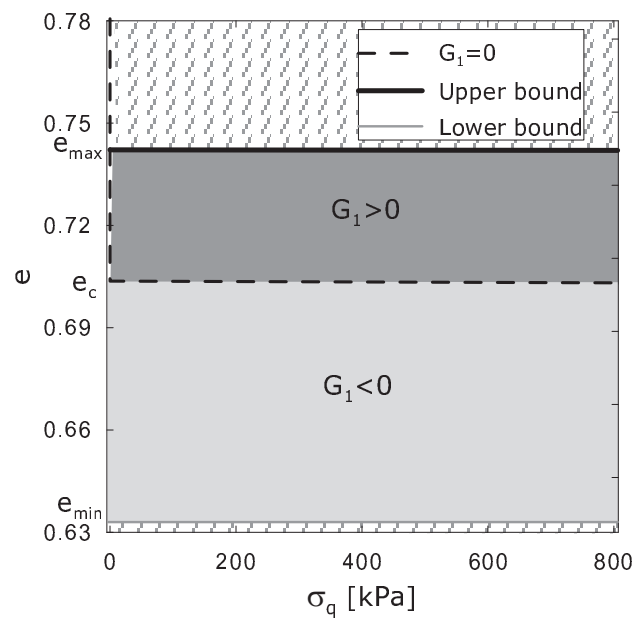

(b)

Figure 5. Admissible states under static conditions in (a) $\tau_{q}-\sigma_{q}$ and (b) $e-\sigma_{q}$ planes.

\section{(ii) Steady state conditions}

With respect to the previous case, where three were the free variables to be imposed to characterize the initial state, the system is here more constrained. In fact, at the steady state, two variables have to be assigned $\left(\dot{\gamma}_{0}\right.$ and either $\sigma_{0}$ or $\left.\tau_{0}\right)$, and four are the constraints to be imposed $\left(\dot{T}=\dot{v}=f=G_{1}=0\right)$.

The initial conditions employed in the following analyses are collected in Table III and are identified by the acronym 'ICX_y_i', where $X$ denotes the type of test: $P$ for constant pressure tests (Section 4.1) and $V$ for constant volume tests (Section 4.2); $y$ indicates the type of initial condition: $s t$ for static conditions and ss for steady state conditions; finally, i represents an identification number. 


\subsection{Constant pressure tests}

In this section, simple shear tests are be simulated by employing a mixed-controlled condition: $\sigma$ and $\dot{\gamma}$ are imposed, whereas $\tau$ and $e$ are measured. In particular, $\sigma$ is kept constant whereas $\dot{\gamma}$ is imposed to vary with time according to the following function:

$$
\dot{\gamma}(t)=\dot{\gamma}_{f}-\left(\dot{\gamma}_{f}-\dot{\gamma}_{0}\right) e^{-t / t_{r}}
$$

where $\dot{\gamma}_{f}$ corresponds to the final shear strain rate asymptotically reached by the material, whereas $t_{r}$ (a sort of characteristic time) governs the evolution of $\dot{\gamma}$ with time. In all the tests numerically simulated here below, $t_{r}=1 \mathrm{~s}$. This implies that after about 7 seconds, the shear strain rate is approximately constant and equal to $\dot{\gamma}_{f}$.

For constant pressure tests, both the two initial conditions are accounted for: static (Section 4.1.1) and steady (Section 4.1.2). In the former case, solely loading tests are analyzed and the influence of $\dot{\gamma}_{f}, e_{0}$ and $\sigma_{0}$ is discussed. In the latter one, for the sake of brevity, one test of unloading is critically discussed. The choice of discussing the influence of $\dot{\gamma}_{f}, \sigma_{0}$ and $e_{0}$ stems from the observation that, according to $\dot{\gamma}_{f}$ and $\sigma_{0}$, the mechanical response of the system may be prevalently either quasi-static

Table III. Initial conditions for constant pressure and constant volume tests.

\begin{tabular}{|c|c|c|c|c|c|}
\hline Code & $\dot{\gamma}_{0}[1 / \mathrm{s}]$ & $T_{0}\left[\mathrm{~m}^{2} / \mathrm{s}^{2}\right]$ & $\tau_{0}$ & $\sigma_{0}[\mathrm{kPa}]$ & $e_{0}$ \\
\hline ICP_st_1 & 0 & $10^{-15}$ & 0 & 50 & 0.66 \\
\hline $\mathrm{IC} P \_s t \_2$ & 0 & $10^{-15}$ & 0 & 50 & 0.70 \\
\hline IC $P \_s t \_3$ & 0 & $10^{-15}$ & 0 & 50 & 0.71 \\
\hline ICP_st_4 & 0 & $10^{-15}$ & 0 & 50 & 0.72 \\
\hline $\mathrm{IC} P \_s t \_5$ & 0 & $10^{-15}$ & 0 & 20 & 0.66 \\
\hline ICP_st_6 & 0 & $10^{-15}$ & 0 & 100 & 0.66 \\
\hline ICP_ss_7 & 2000 & 0.773 & 23.02 & 50 & 0.70356 \\
\hline IC $V \_s t \_1$ & 0 & $10^{-15}$ & 0 & 50 & 0.7034 \\
\hline $\mathrm{IC} V \_s t \_2$ & 0 & $10^{-15}$ & 0 & 50 & 0.7035 \\
\hline IC $V \_s t \_3$ & 0 & $10^{-15}$ & 0 & 50 & 0.70356 \\
\hline IC $V \_s t \_4$ & 0 & $10^{-15}$ & 0 & 50 & 0.7037 \\
\hline IC $V \_s t \_5$ & 0 & $10^{-15}$ & 0 & 100 & 0.7035 \\
\hline IC $V \_s t \_6$ & 0 & $10^{-15}$ & 0 & 200 & 0.7035 \\
\hline
\end{tabular}


or collisional, whereas both $e_{0}$ and $\sigma_{0}$ influence the volumetric response of the material throughout the definition of the plastic potential (Equation 21).

\subsubsection{From static initial conditions}

\section{Influence of $\dot{\gamma}_{\mathrm{f}}$}

All the tests discussed in this sub-section refer to the initial condition ICP_st_1 of Table III and refer to a dense ideal material for which $G_{1}<0$ at $t=t_{0}$. The six tests numerically performed differ from each other for $\dot{\gamma_{f}}\left(\right.$ Test 1: $\dot{\gamma}_{f}=1001 / \mathrm{s}$, Test $2: \dot{\gamma}_{f}=10001 / \mathrm{s}$, Test $3: \dot{\gamma_{f}}=20001 / \mathrm{s}$, Test 4: $\dot{\gamma}_{f}=30001 / \mathrm{s}$, Test 5: $\dot{\gamma}_{f}=4000$ 1/s, Test $\left.6: \dot{\gamma}_{f}=60001 / \mathrm{s}\right)$. For the sake of clarity, in all the figures reported below, the points corresponding to both the initial and the final states are denoted with $I_{i}$ and $S_{i}$, respectively, where subscript $i$ indicates the test number. The obtained results (Figure 6) clearly show that:

- initially and independently of $\dot{\gamma}_{f}$, the mechanical response is almost elastic (pseudo-vertical straight line in Figure 6a).

- During a second phase and according to the imposed $\dot{\gamma}_{f}$, the stress path in the $\tau_{q}-\sigma_{q}$ plane reaches final values of $\sigma_{q}$ and $\tau_{q}$ progressively smaller all belonging to the $f=0$ locus (dashed line in Figure 6a); in case $\dot{\gamma}_{f}$ is sufficiently large (Tests 5 and 6), the origin of the axes is got and the phase transition to the collisional regime takes place.

- All the tests are performed until the steady state is reached. This is evident from Figures 6c, $6 \mathrm{~d}, 6 \mathrm{e}$ and $6 \mathrm{f}$.

- By increasing $\dot{\gamma}_{f}$, a more dilatant response is obtained (Figure 6b). In two cases (Tests 5 and 6) the void ratio increases over $e_{\max }$. To clearly visualize the paths, the graph in Figure $6 \mathrm{~b}$ has been cut in correspondence of $e=e_{\max }$, therefore the final point $\mathrm{S}_{5}$ and $\mathrm{S}_{6}$ are not reported. In the $e-\sigma_{q}$ plane, the pseudo-elastic initial phases degenerate into the initial points $\left(I_{1}=I_{2}=I_{3}=I_{4}=I_{5}=I_{6}\right)$. All paths refer to the second phase for which $f=\dot{f}=0$. By changing $\dot{\gamma}_{f}$, the critical state locus $G_{1}=0$ (dashed line) is achieved for different values of $e$ and $\sigma_{q}$.

- From Figures 6a and 6b, it is evident that paths of Tests 1, 2, 3, and 4 pass throughout regimes (I), (II), and (III) of Figure 4, whereas paths of Tests 5 and 6 pass throughout regimes (I), (II), (III) and (IV), getting finally the collisional steady state. 
- The change in the void ratio evolution is particularly evident in case the material reaches the pure collisional state (Tests 5 and 6 in Figure 6c). The nullification of the quasi-static contribution is in this figure testified by the rapid step-wise increase in the void ratio.

- By increasing $\dot{\gamma}_{f}$, the collisional stresses increase, and in case $\dot{\gamma}_{f}$ is sufficiently large (Tests 5 and 6), a peak in both the $\tau_{c}-\gamma$ and $\tau-\gamma$ planes is present (Figures 6e and 6f, respectively).

- Exclusively in case $\dot{\gamma}_{f}$ is sufficiently small (Test 1), the $\tau-\gamma$ response seems to be perfect elasto-plastic (Figure 6f).

\section{Influence of $e_{0}$}

Figure 7 refers to four numerical tests performed by imposing different values of $e_{0}$ (Test 1 : ICP_st_1, Test 2: ICP_st_2, Test 3: ICP_st_3 and Test 4: ICP_st_4) and $\dot{\gamma}_{f}=2000$ 1/s. The value of $e_{0}$ affects the way in which the volume varies before the attainment of the steady state. Independently of the value of $e_{0}$, the paths reach the same final stationary condition. In the $\tau_{q}-\sigma_{q}$ and $e-\sigma_{q}$ planes (Figures $7 \mathrm{a}$ and $7 \mathrm{~b}$ ), paths start from different points $\left(I_{1}, I_{2}, I_{3}, I_{4}\right)$ pass throughout regimes (I), (II) and (III) of Figure 4 and arrive to the same steady state belonging to the critical state locus $\left(S_{1}=S_{2}=S_{3}=S_{4}\right)$. In particular, the paths are perfectly coincident in the $\tau_{q}-\sigma_{q}$ plane (Figure 7a), while the evolution of the void ratio differs significantly (Figure 7b). The initially dense materials (Tests 1 and 2), reach the $G_{1}=0$ locus by dilating. The initially loose materials (Tests 3 and 4) initially dilate but, subsequently, compact until the attainment of the ultimate point (see also Figure 7c). Graphs illustrating the variation of $T, \tau_{c}$ and $\tau$ against the shear strain $\gamma$, are negligibly affected by the initial void ratio and, for the sake of brevity, they are not here reported.

\section{Influence of $\sigma_{0}$}

The results here considered refer to three tests performed by imposing three different values of $\sigma_{0}$ (Test 1: ICP_st_5, Test 2: ICP_st_1, Test 3: ICP_st_6) and $\dot{\gamma}_{f}=2000$ 1/s. Unless the different value of $\sigma_{0}$ imposed, owing to the $e_{0}$ assumed, in all the three cases the material points at the beginning markedly dilate (Figures $8 \mathrm{~b}$ and $8 \mathrm{c}$ ). As is illustrated in Figures $8 \mathrm{a}$ and $8 \mathrm{~b}$, the $\tau_{q}-\sigma_{q}$ and $e-\sigma_{q}$ paths start from different initial states $\left(I_{1}, I_{2}\right.$ and $\left.I_{3}\right)$ and reach distinct final points lying on the critical state locus $\left(S_{1}, S_{2}\right.$ and $\left.S_{3}\right)$. In particular, paths of Tests 2 and 3 pass throughout regimes (I), (II) and (III) of Figure 4, whereas the path of Test 1 passas throughout regimes (I), (II), (III) and (IV). Indeed, for decreasing values of the imposed normal stress, quasi-static stresses decrease; when $\sigma_{0}$ is sufficieltly small (Test 1 ), the corresponding $\tau_{q}-\sigma_{q}$ path reaches the origin of the axes: total stresses coincide with collisional stresses and the material reaches the collisional steady state 


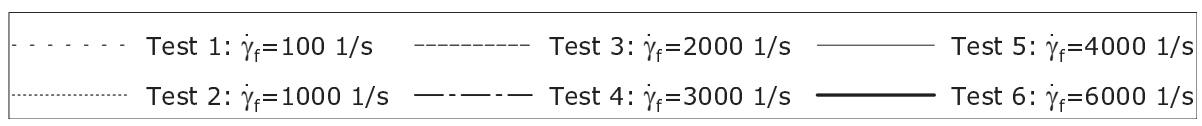

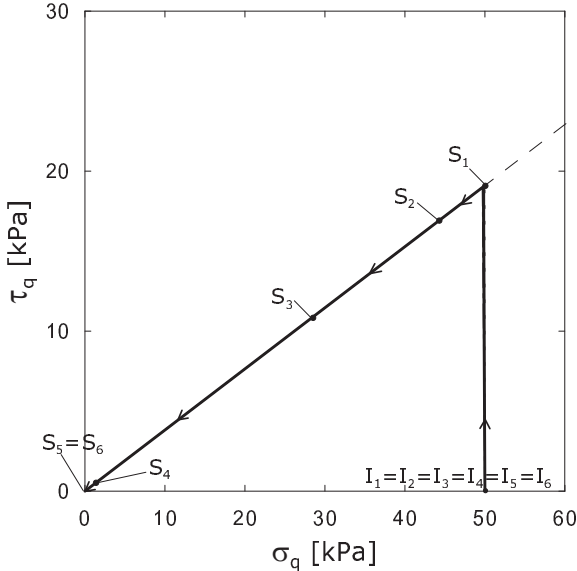

(a)

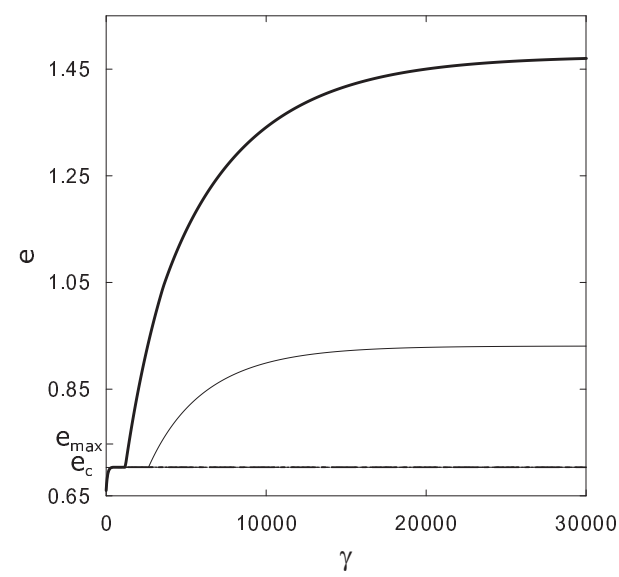

(c)

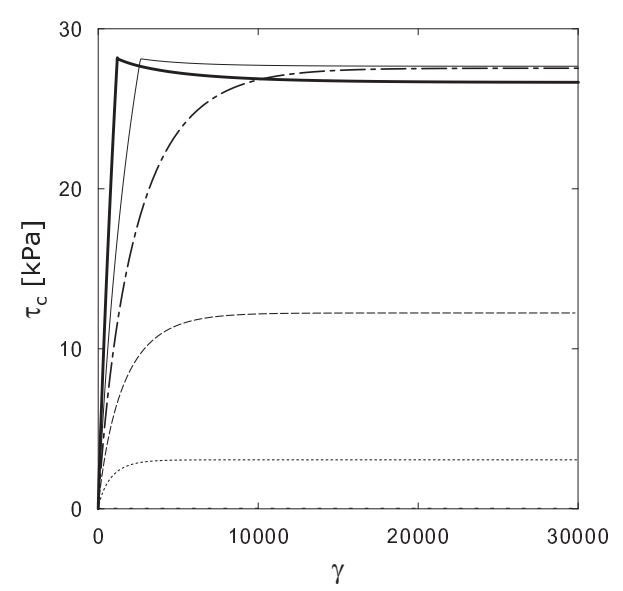

(e)

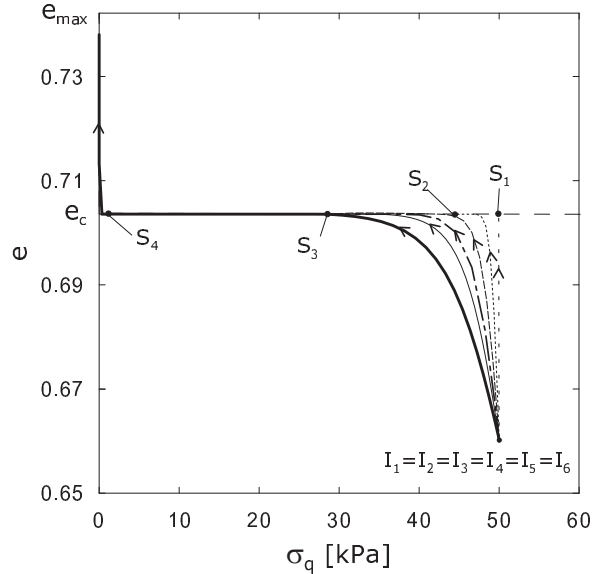

(b)

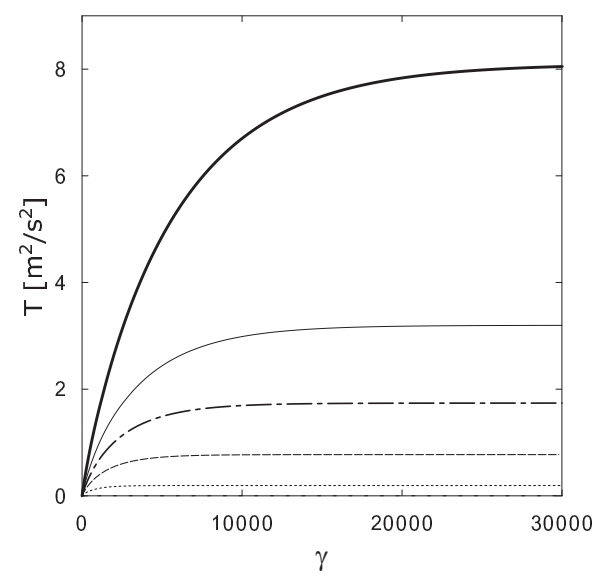

(d)

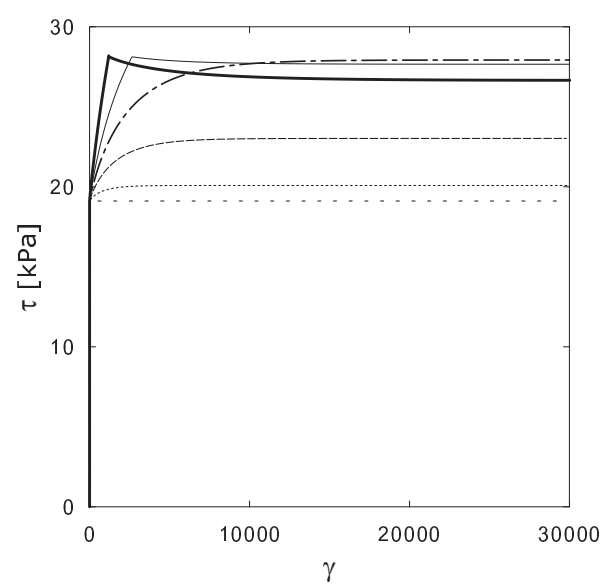

(f)

Figure 6. Influence of $\dot{\gamma}_{f}$ for constant pressure tests relative to a dense material (ICP_st_1) on the planes: (a) $\tau_{q}-\sigma_{q}$, (b) $e-\sigma_{q}$, (c) $e-\gamma$, (d) $T-\gamma$, (e) $\tau_{c}-\gamma$, (f) $\tau-\gamma$. 
(Figure 8a). Analogously to what observed in Figure 6 for very high shear rates, when force chains disappear, a rapid increase in the void ratio (Figure 8c) occurs. In the three tests here considered, the total shear stress increases monotonically (Figure $8 \mathrm{f}$ ). Since the $\dot{\gamma}$ imposed coincides, the granular temperature evolution seems to coincide too (Figure 8d).

\subsubsection{From steady initial conditions}

In this sub-section, exclusively the numerical results of an unloading test are discussed. At time $t_{0}$ the material is assumed to flow under steady conditions (ICP_ss_4). The test is characterized by a continuous decrease in $\dot{\gamma}$, until an asymptotic value of $\dot{\gamma}_{f}=-20001 / \mathrm{s}$. The numerical results are

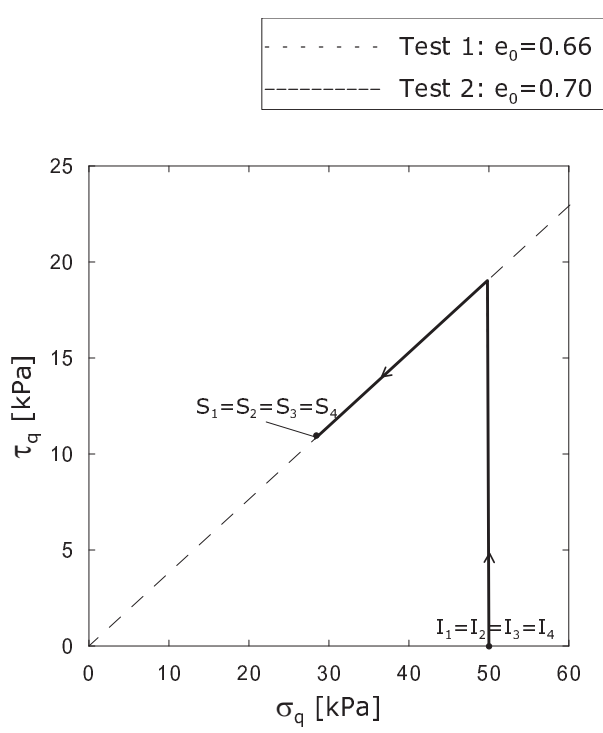

(a)
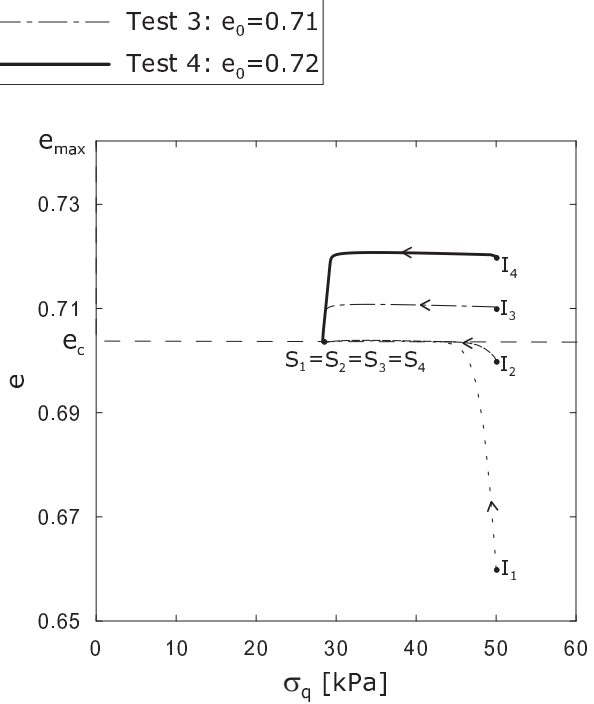

(b)

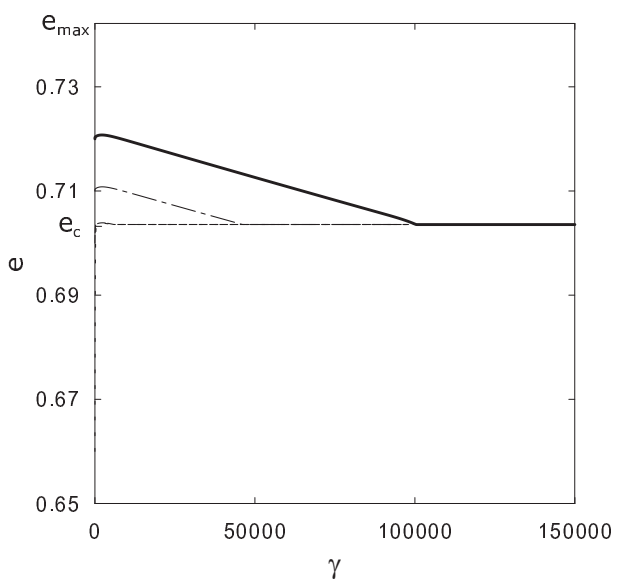

(c)

Figure 7. Influence of $e_{0}$ for constant pressure tests on the planes: (a) $\tau_{q}-\sigma_{q}$, (b) $e-\sigma_{q}$, (c) $e-\gamma$. 


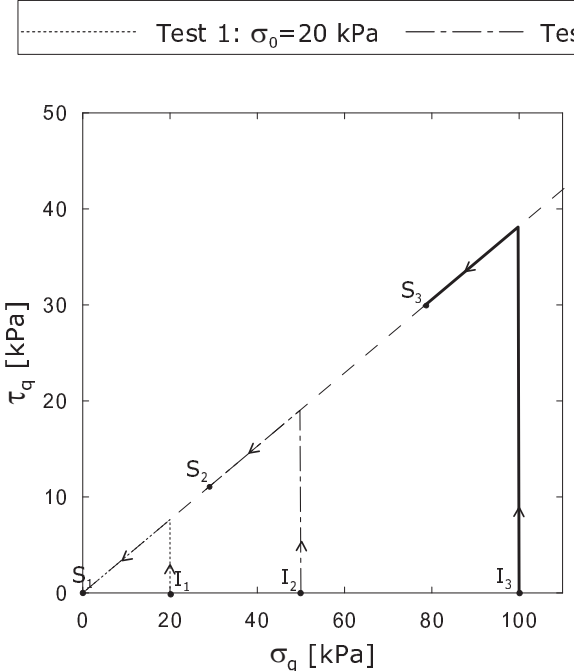

(a)

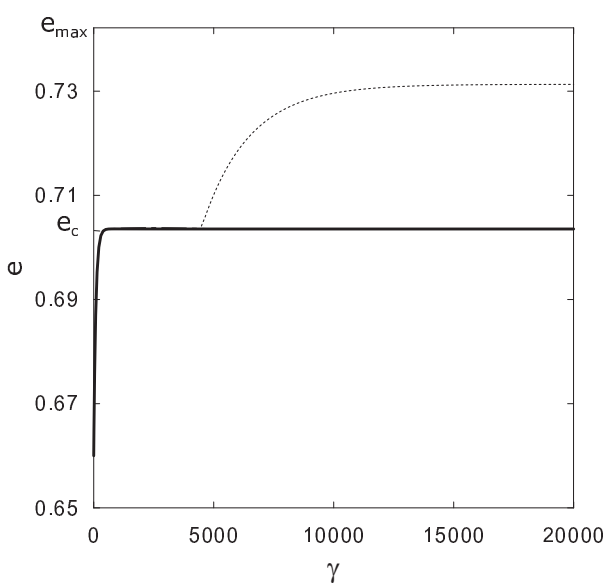

(c)

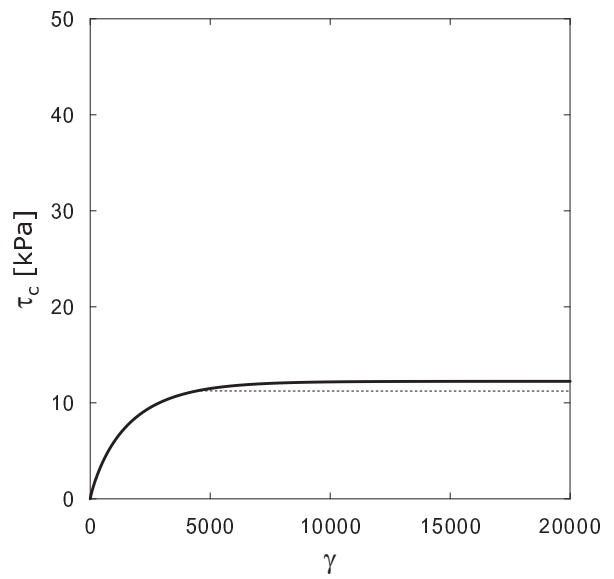

(e)

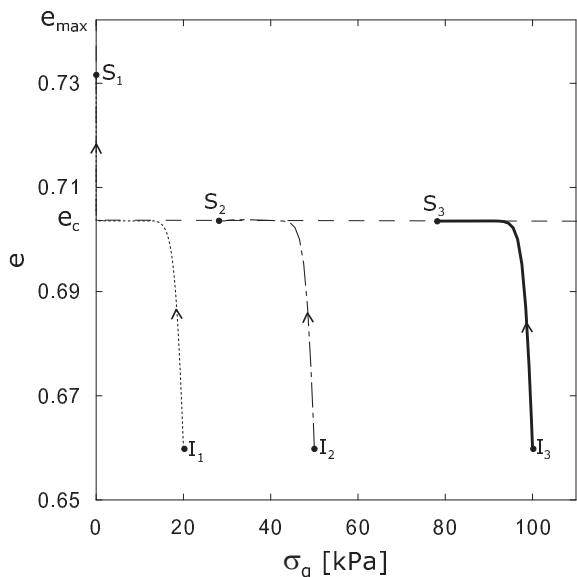

(b)

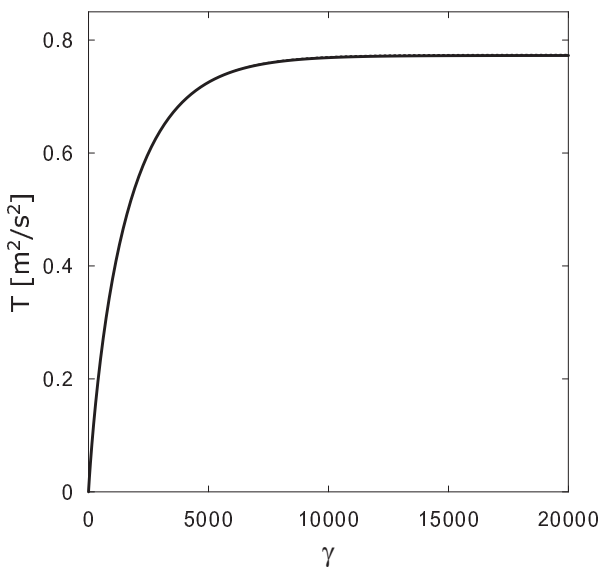

(d)

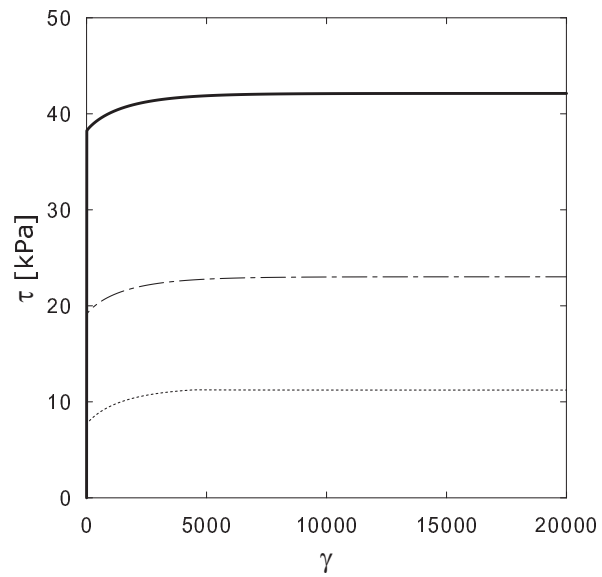

(f)

Figure 8. Influence of $\sigma_{0}$ for constant pressure tests on the planes: (a) $\tau_{q}-\sigma_{q}$, (b) $e-\sigma_{q}$, (c) $e-\gamma$, (d) $T-\gamma$, (e) $\tau_{c}-\gamma$, (f) $\tau-\gamma$. 
reported in Figure 9. The test can be subdivided in 3 phases, corresponding to three regimes of Figure 4:

- initially, from $I$ to $C$, the material flows by satisfying conditions $f=0$ and $G_{1}=0$ (regime (III)) and $\dot{\gamma}>0$. At point $C, \dot{\gamma}$ nullifies. Quasi-static stresses increase (Figures 9a and 9b) while all the remaining stress variables decrease and $\tau_{c}$ rapidly vanishes (Figures $9 \mathrm{e}$ and 9f). As was expected, even $\mathrm{T}$ rapidly reduces until its nullification (Figure 9d);

- from $C$ to $Y$, the material enters into the visco-elastic regime $(f<0$, regime (I)) and $\dot{\gamma}<0$. At point $Y$, the material yields again $\left(f=0, G_{1} \neq 0\right.$, regime (II)). Both $T$ and $\tau_{c}$ remain negligible (Figures $9 \mathrm{~d}$ and $9 \mathrm{e}$ ). At point $M$, both $\tau$ and $\tau_{c}$ become negative;

- from $Y$ to $S$ (corresponding this latter to the final steady state), $G_{1}>0$ and $f=0$ (regime (II)). Again the void ratio changes very slightly until condition $G_{1}=0$ is newly satisfied (regime (III)). At the end of the test, stationary conditions are achieved.

\subsection{Constant volume tests}

The numerical results are in this case obtained by imposing a constant void ratio $\left(e=e_{0}\right)$ and by changing $\dot{\gamma}$ according to Equation 50, while $\sigma$ and $\tau$ are measured. Conversely to Section 4.1, solely static conditions are here considered. In Table III, the initial static conditions are listed. As was done in sub-section 4.1.1, hereafter the influence of $\dot{\gamma}_{f}, e_{0}$ and $\sigma_{0}$ on the numerical results is analyzed separately.

\section{Influence of $\dot{\gamma}_{\mathrm{f}}$}

The results here presented are obtained by imposing ICV_st_2 of Table III. The six tests numerically performed differ to each other for $\dot{\gamma}_{f}$ : Test 1: $\dot{\gamma}_{f}=100$ 1/s, Test 2: $\dot{\gamma}_{f}=1000$ 1/s, Test 3: $\dot{\gamma}_{f}=2000$ 1/s, Test 4: $\dot{\gamma}_{f}=30001 / \mathrm{s}$, Test 5: $\dot{\gamma}_{f}=40001 / \mathrm{s}$ and Test $6: \dot{\gamma}_{f}=60001 / \mathrm{s}$. As is evident from Figures 10a and 10b, when the initial void ratio is coincident, the response of the model in terms of $\tau_{q}-\sigma_{q}$ and $e-\sigma_{q}$ coincides, independently of the value of $\dot{\gamma}_{f}$ imposed. Since initially $G_{1}<0$ (dense case), the $\tau_{q}-\sigma_{q}$ curves, as was expected, are characterized by a monotonic increasing trend (Figure 10a). The granular temperature is severely affected by the value of $\dot{\gamma}_{f}$ imposed (Figure 10c). This is also testified by the response in terms of $\sigma$ and $\tau$ (Figures 10e and 10f). The stress ratio $\tau / \sigma$ is equal to $\tan \phi^{\prime}$ only in case $\dot{\gamma}_{f}$ is sufficiently small (Test 1 of Figure 10d). All the paths pass throughout regimes (I), (II) and (III) of Figure 4. 


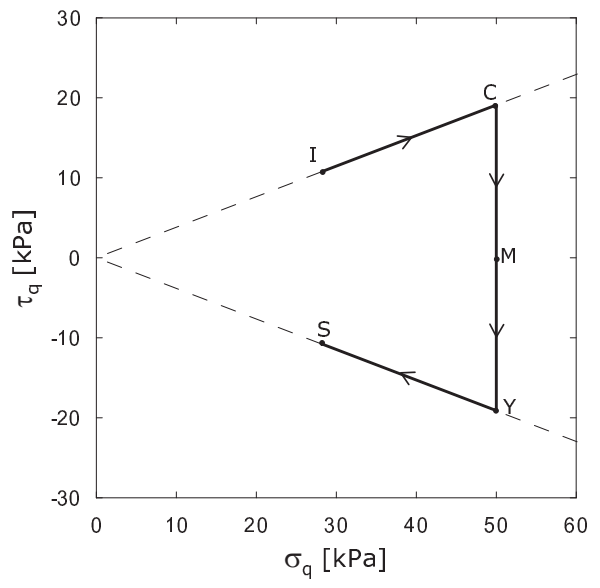

(a)

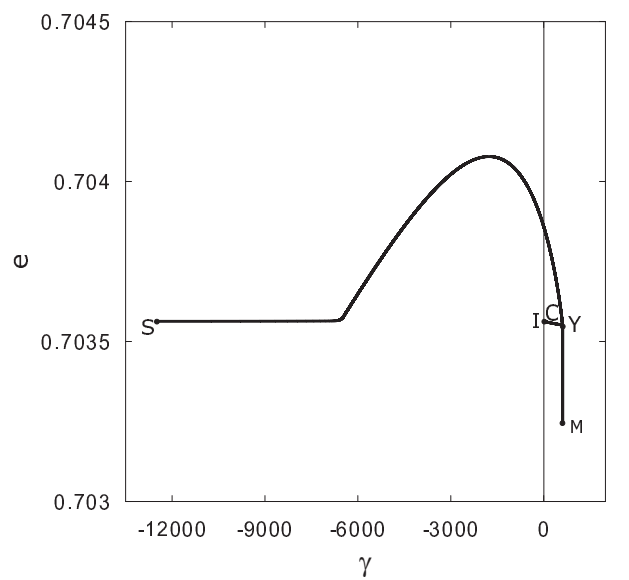

(c)

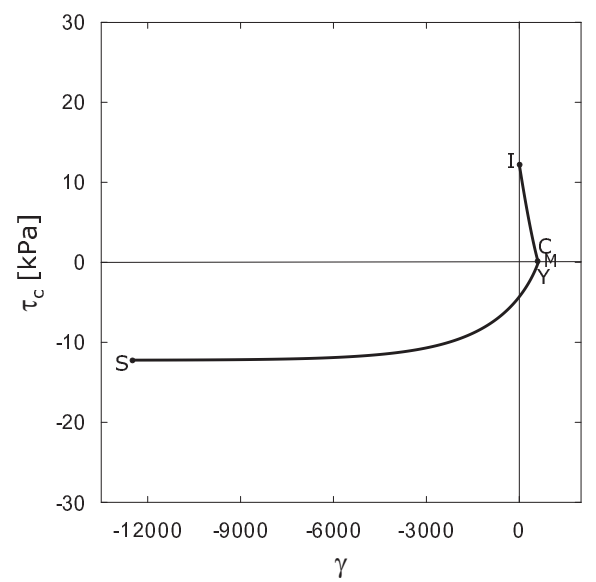

(e)

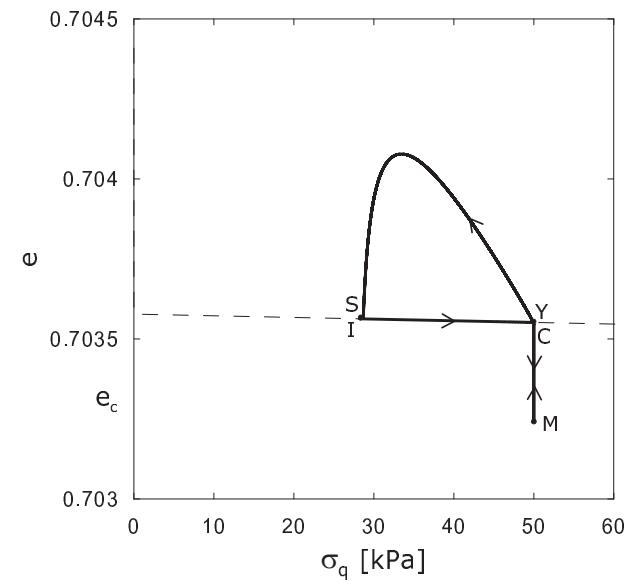

(b)

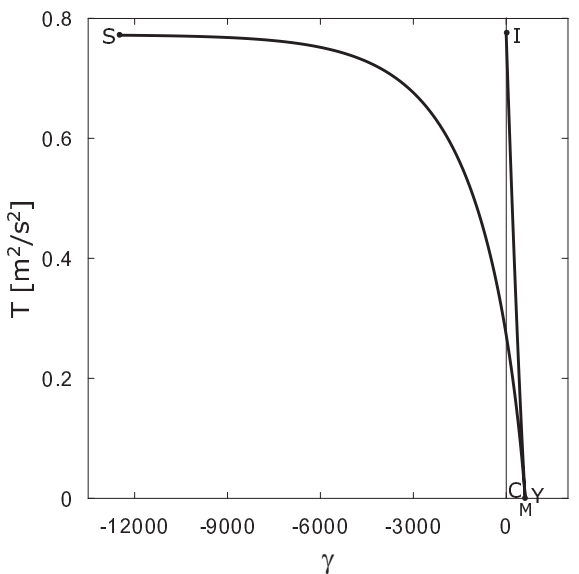

(d)

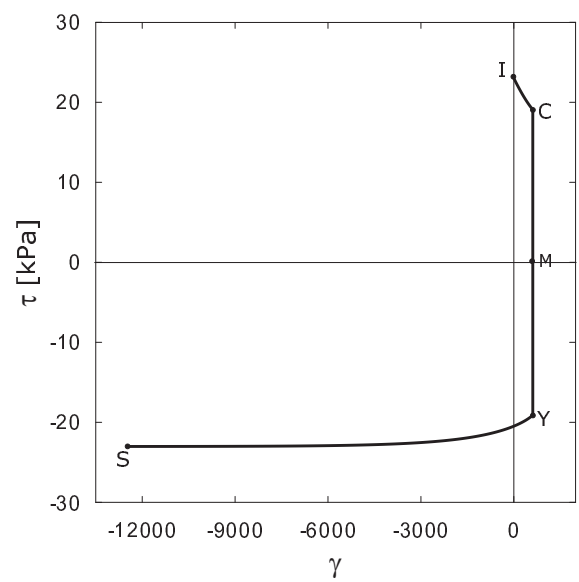

(f)

Figure 9. Numerical response for a constant pressure test relative to a material initially flowing under steady state conditions (ICP_ss_4) and $\dot{\gamma}_{f}=-2000$ 1/s on the planes: (a) $\tau_{q}-\sigma_{q}$, (b) $e-\sigma_{q}$, (c) $e-\gamma$, (d) $T-\gamma$, (e) $\tau_{c}-\gamma$, (f) $\tau-\gamma$. 


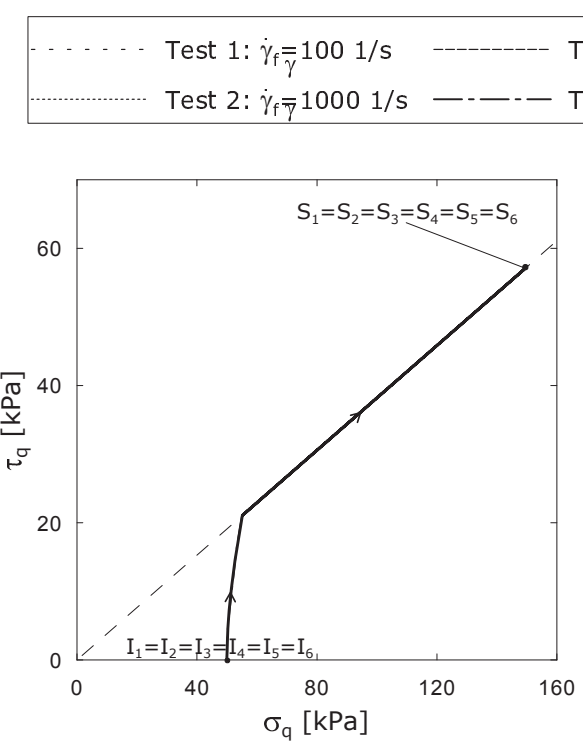

(a)

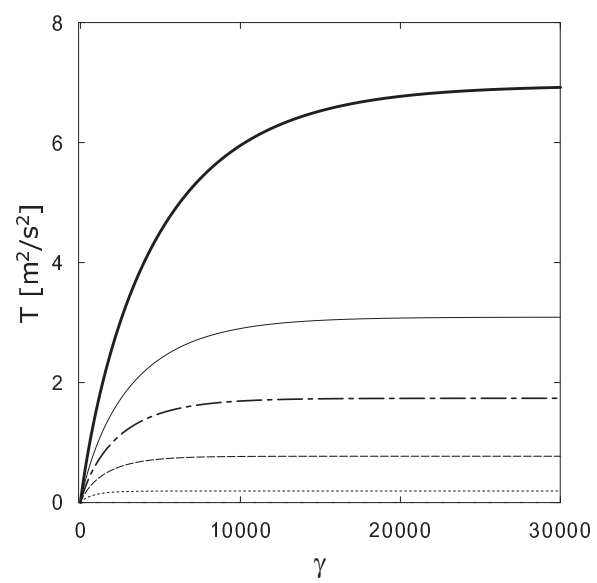

(c)

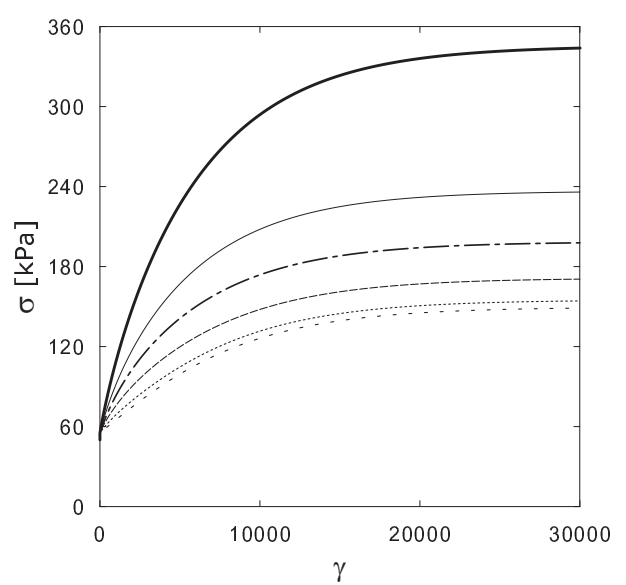

(e)
Test 3: $\dot{\gamma}_{\mathrm{f}}=20001 / \mathrm{s}$

Test 4: $\dot{\gamma}_{\mathrm{f}}=30001 / \mathrm{s}$

Test 5: $\dot{\gamma}_{\mathrm{f}}=40001 / \mathrm{s}$

Test 6: $\dot{\gamma}_{\mathrm{f}}=60001 / \mathrm{s}$

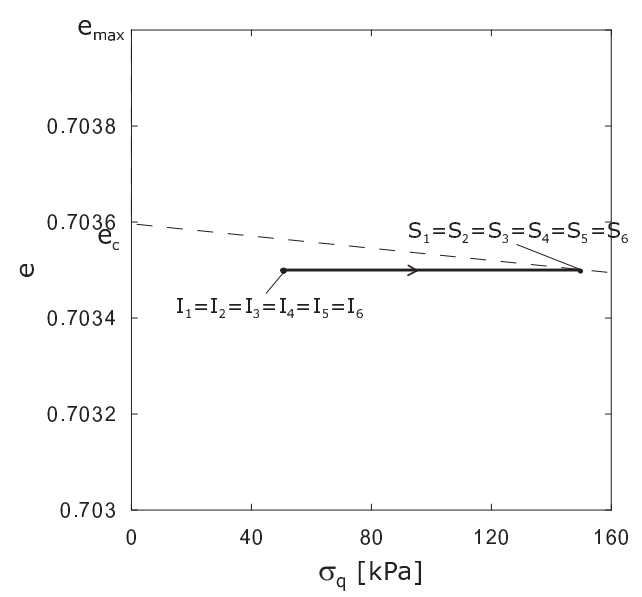

(b)

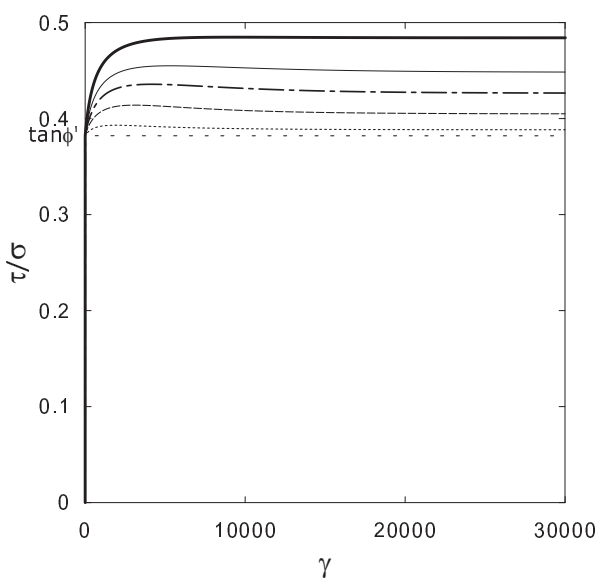

(d)

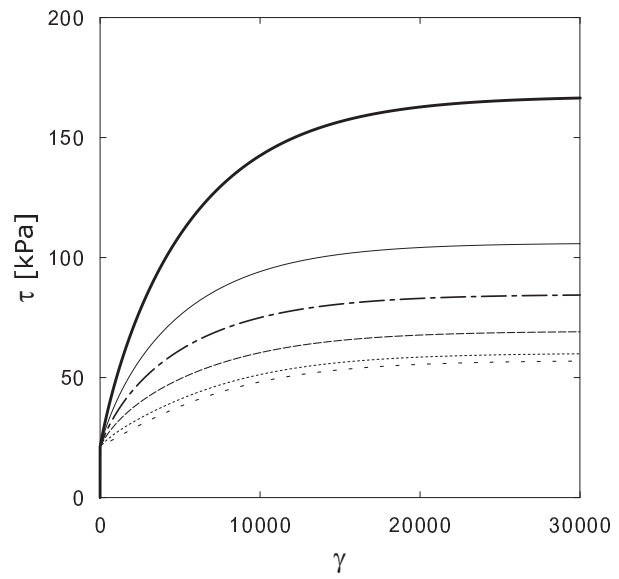

(f)

Figure 10. Influence of $\dot{\gamma}_{f}$ for constant volume tests relative to a dense material (ICV_st_2) on the planes: (a) $\tau_{q}-\sigma_{q}$, (b) $e-\sigma_{q}$, (c) $T-\gamma$, (d) $\tau / \sigma-\gamma$, (e) $\sigma-\gamma$, (f) $\tau-\gamma$. 


\section{Influence of $e_{0}$}

In this case, different $e_{0}$ values are imposed, while $\dot{\gamma}_{f}=20001 / \mathrm{s}$. Four different tests were performed: Test 1: ICV_st_1, Test 2: ICV_st_2, Test 3: IC $V_{-} s t_{-} 3$ and Test 4: IC $V_{-} s t_{-} 4$. The $\tau_{q}-\sigma_{q}$ and $e-\sigma_{q}$ paths, although starting from different initial void ratios $e_{0}$, all get the critical state (Figures 11a and 11b): at increasing values of $e_{0}$, the final $\sigma_{q}, \tau_{q}$ values decrease. In case $e_{0} \geq e_{c}$ (Test 4), both $\sigma_{q}$ and $\tau_{q}$ nullify. Then, path of Test 4 passes throughout regimes (I), (II) and (IV) of Figure 4, whereas paths of Tests 1, 2 and 3 pass throughout regimes (I), (II) and (III). The $\tau / \sigma$ ratio evolution, as well as the $\tau, \sigma$ evolutions, is markedly affected by $e_{0}$ (Figures 11d-11f). In case of "dense" specimens (Tests 1 and 2), the response in terms of $\tau / \sigma, \sigma$ and $\tau$ is pseudo-hardening; in case of "loose" specimens (Tests 3 and 4), a pseudo-softening takes place (Figures 11e and 11f). In contrast, since $\dot{\gamma}_{f}$ is kept constant, in the $T-\gamma$ plane all the curves differ very slightly (Figure 11c). When $e_{0}$ is sufficiently large, $\tau / \sigma$ increases over $\tan \phi^{\prime}$ (Tests 2, 3, and 4 of Figure 11d).

\section{Influence of $\sigma_{0}$}

The results here considered refer to three tests performed by imposing three different $\sigma_{0}$ values (Test 1: ICV_st_2, Test 2: ICV_st_5 and Test 3: ICV_st_6) and $\dot{\gamma}_{f}=20001 / \mathrm{s}$. Since both $e_{0}$ and $\dot{\gamma}_{f}$ coincide for the three ideal numerical tests, the final steady states reached coincide $\left(S_{1}=S_{2}=S_{3}\right)$, whereas the stress paths in the $\tau_{q}-\sigma_{q}$ and $e-\sigma_{q}$ planes totally differ (Figures 12a and 12b), although all the paths pass throughout regimes (I), (II) and (III). The curves in the $T-\gamma$ plane (Figure 12c) perfectly coincide, therefore the difference in $\tau / \sigma, \sigma$ and $\tau$ derive solely from the different response in the $\tau_{q}-\sigma_{q}$ plane (Figures 12d, 12e and 12f). Once again, the pseudo-softening is evident only in case of loose initial conditions (Test 3).

\section{CONCLUDING REMARKS}

In this paper, a constitutive model capable of simulating both the inception and the post-collapse behaviour of granular matters under simple shear conditions has been proposed. According to the conceived constitutive relationship, stresses are calculated as the sum of two contributions: a quasi-static and a collisional one. The former one accounts for the response of the granular matter as a solid-like continuum, the latter one for its fluid-like nature. The quasi-static contribution is calculated by assuming a perfect elasto-plastic constitutive relationship, whereas the collisional contribution is modelled according to the kinetic theory of granular gases. The transition form solidlike to fluid-like conditions is therefore assumed to be governed by the granular temperature and 


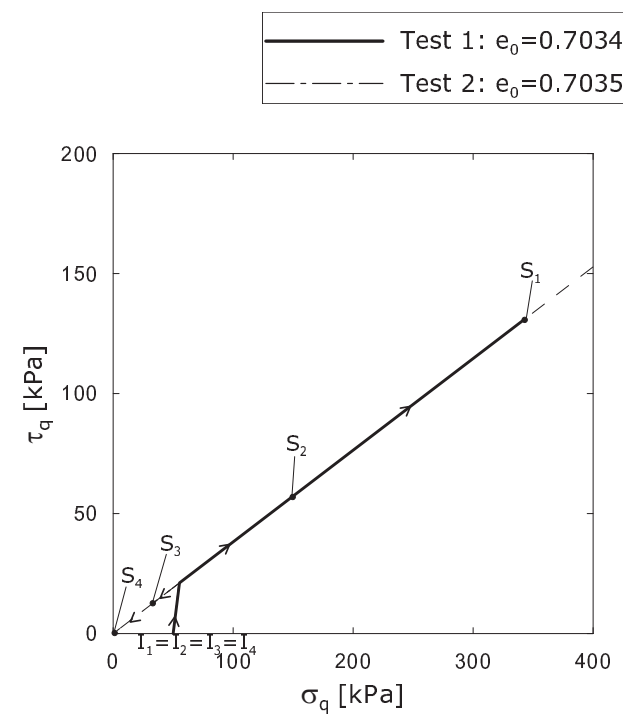

(a)

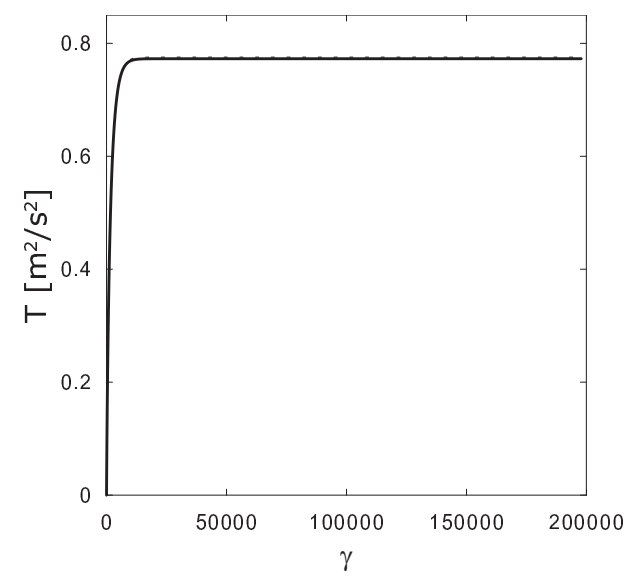

(c)

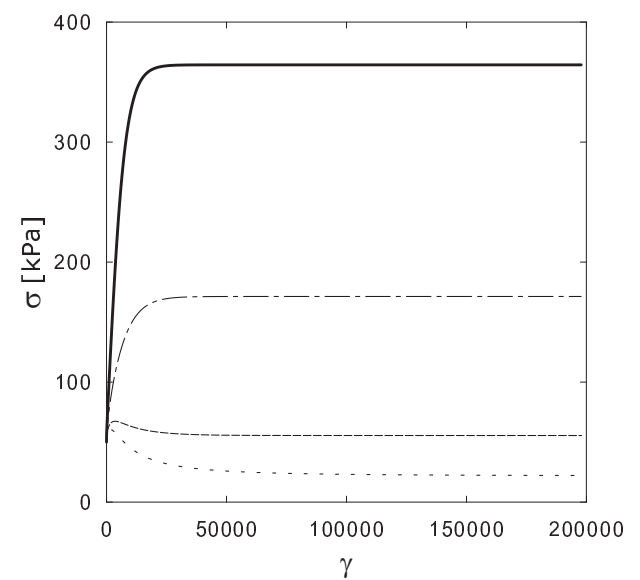

(e)
------- Test $3: e_{0}=0.70356$
$\ldots-$ Test $4: e_{0}=0.7037$

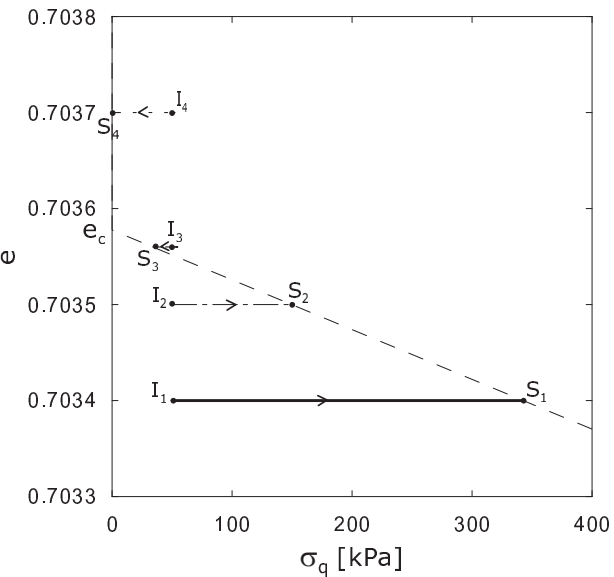

(b)

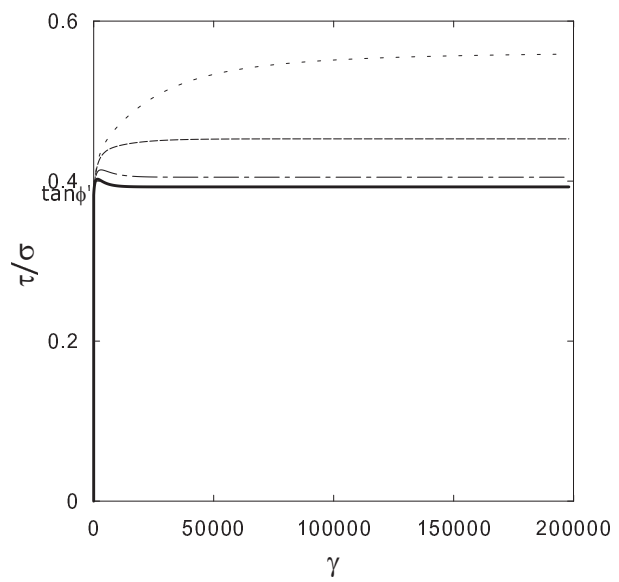

(d)

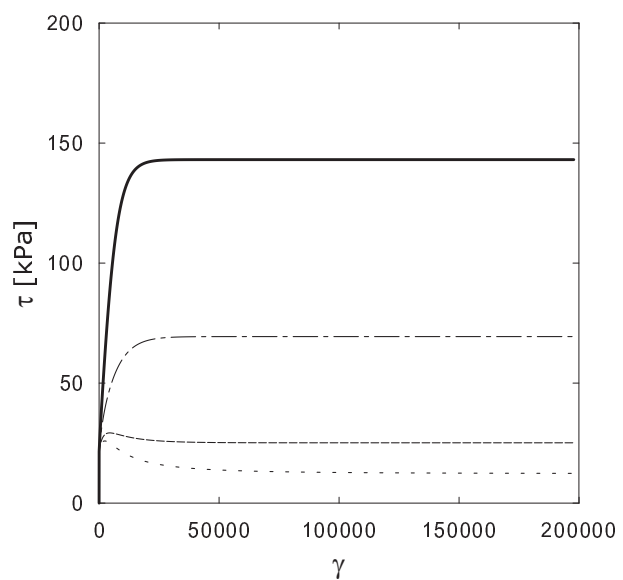

(f)

Figure 11. Influence of $e_{0}$ for constant volume tests on the planes: (a) $\tau_{q}-\sigma_{q}$, (b) $e-\sigma_{q}$, (c) $T-\gamma$, (d)

$$
\tau / \sigma-\gamma,(\mathrm{e}) \sigma-\gamma, \text { (f) } \tau-\gamma \text {. }
$$




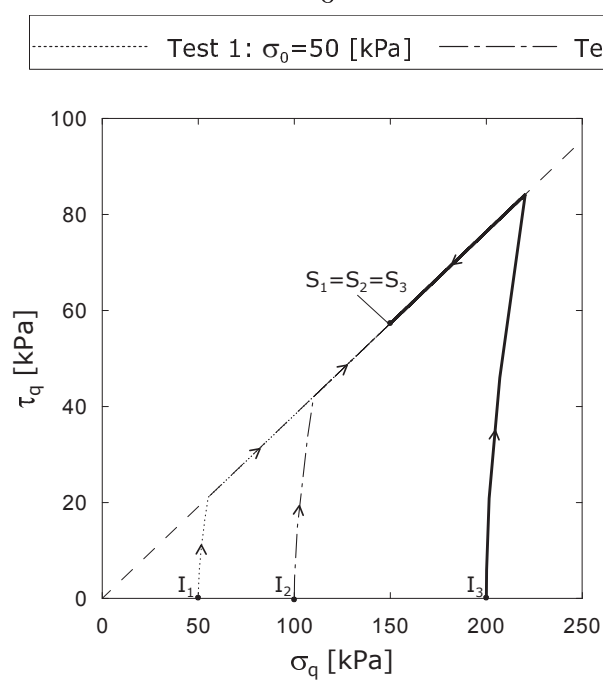

(a)

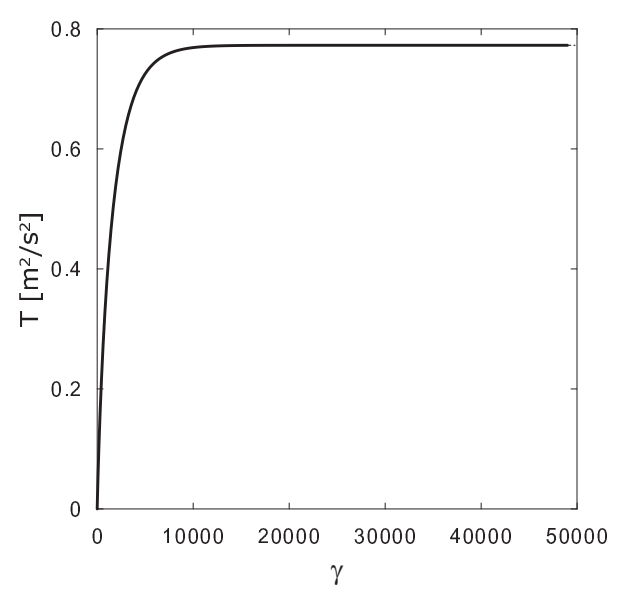

(c)

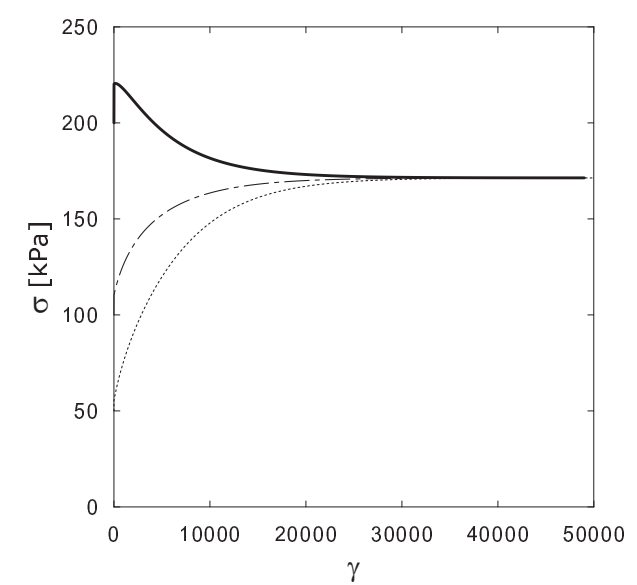

(e)

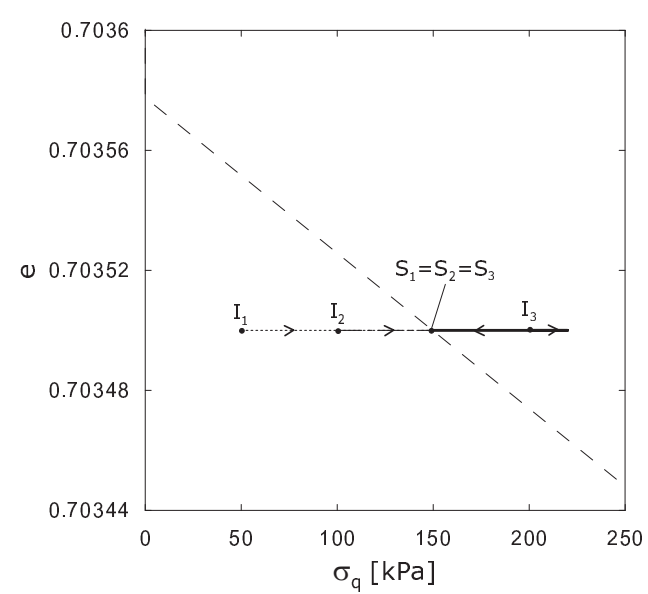

(b)

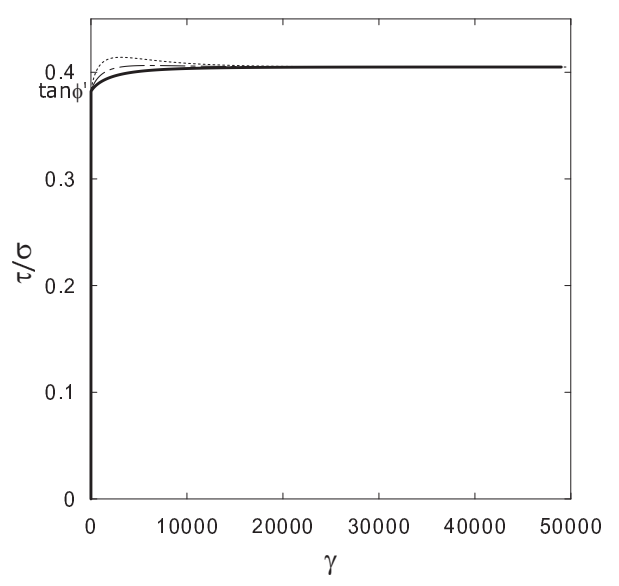

(d)

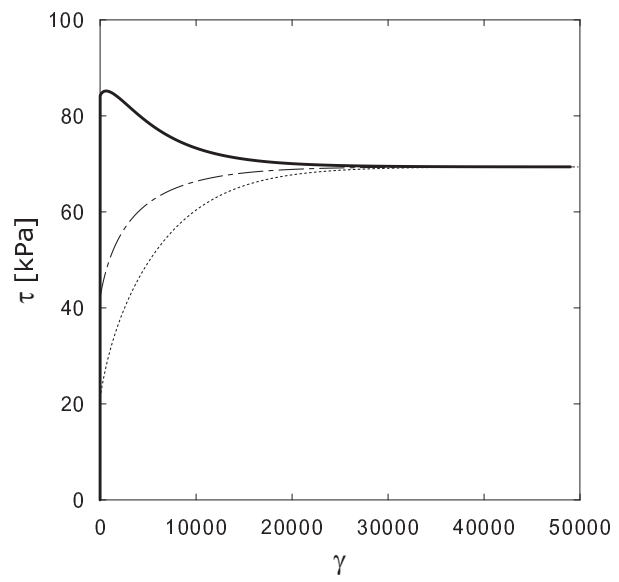

(f)

Figure 12. Influence of $\sigma_{0}$ for constant volume tests on the planes: (a) $\tau_{q}-\sigma_{q}$, (b) $e-\sigma_{q}$, (c) $T-\gamma$, (d) $\tau / \sigma-\gamma,(\mathrm{e}) \sigma-\gamma$, (f) $\tau-\gamma$. 
the void ratio, the unique state variables of the model. Constitutive equations have been integrated in time by considering two different tests: constant pressure and constant volume. Both loading and unloading tests have been simulated. Admissible initial conditions have been imposed in order to reproduce either an initially static system or a system flowing under stationary conditions. The model is based on the definition of four distinct unsteady regimes: the visco-elastic, the visco-elastoplastic, the critical and the collisional. The transition from one regime to another is governed by the fulfillment of suitable mechanical conditions.

The numerical results testify the capability of the model of taking into account the dependence of the mechanical behaviour of granular matters on the initial void ratio, the imposed normal pressure and, in particular, the imposed shear strain rate.

The originality of the approach proposed derives from the interpretation of the critical state as a peculiar steady state taking place for the granular temperature approaching a zero value, i.e. for quasi-static conditions. Under unsteady conditions, the authors introduce a critical regime, where the void ratio evolution is governed by the normal quasi-static stress via the critical state locus definition. This implies that the plastic volumetric strain rate does not obey the flow-rule but is computed by depurating the total volumetric strain rate of the elastic component.

Further extensions of the theoretical framework here suggested to three dimensional conditions and to strain-hardening plasticity are remanded to subsequent studies.

\section{REFERENCES}

1. Calvetti F, Crosta G, Tatarella M. Numerical simulation of dry granular flows: from the reproduction of small-scale experiments to the prediction of rock avalanches. Rivista Italiana di Geotecnica 2000; 2: 21-38.

2. Pastor M, Haddad B, Sorbino G, Cuomo S, Drempetic P. A depth-integrated, coupled SPH model for flow-like landslides and related phenomena. International Journal for Numerical and Analytical Methods in Geomechanics 2009; 33: 143-172.

3. Andersen S, Andersen L. Modelling of landslides with the material-point method. Computers \& Geosciences 2010; 14: 137-147.

4. Cremonesi M, Frangi A, Perego U. A Lagrangian finite element approach for the simulation of water-waves induced by landslides. Computers and Structures 2011; 89(11-12): 10861093.

5. Prime N, Dufour F, Darve F. Solid-fluid transition in geometarials and application to a mudflow interacting with an obstacle. International Journal for Numerical and Analytical Methods in Geomechanics 2014; 38(13): 1341-1361.

6. Pirulli M, Bristeau MO, Mangeney A, Scavia C. The effect of the earth pressure coefficients on the runout of granular material. Environmental Modelling \& Software 2007; 22: 1437-1454.

7. Faccanoni G, Mangeney A. Exact solution for granular flows. International Journal for Numerical and Analytical Methods in Geomechanics 2012; 37: 1408-1433. 
8. Jaeger HM, Nagel SM, Behringer RP. Granular solids, liquids, and gases. Reviews of Modern Physics 1996; 68: 12591273.

9. Campbell CS. Granular material flows An overview. Powder Technology 2006; 162: 208-229.

10. Forterre Y, Pouliquen O. Flows of dense granular media. Annual Review of Fluid Mechanics 2008; 40: 124.

11. Jenkins JT, Savage SB. A theory for the rapid flow of identical, smooth, nearly elastic, spherical particles. Journal of fluid mechanics 1983; 130: 187202.

12. Savage SB. The mechanics of rapid graular flows. Advances in Applied Mechanics 1984; 24: 289-366.

13. Campbell CS. Rapid granular flows. Annual Review of Fluid Mechanics 1990; 22: 5792.

14. Lun CKK. Kinetic theory for granular flow of dense, slightly inelastic, slightly rough spheres. Journal of fluid mechanics 1991; 223: 539559.

15. Garzó V, Dufty JW. Dense fluid transport for inelastic hard spheres. Physical Review E 1999; 59: 5895-5911.

16. Goldhirsch I. Rapid granular flows. Annual Review of Fluid Mechanics 2003; 35: 267293.

17. Jenkins JT, Richman MW. Grad's 13-moment system for a dense gas of inelastic spheres. Archive for Rational Mechanics and Analysis 1985; 87: 355377.

18. Lun CKK, Savage SB. A simply kinetic theory for granular flow of rough, inelastic, spherical particles. Journal of applied mechanics 1987; 54: 4753.

19. Goldstein A, Shapiro M. Mechanics of collisional motion of granular materials. Part 1. General hydrodynamic equations. Journal of Fluid Mechanics 1995; 282: 75114.

20. Jenkins JT, Zhang C. Kinetic theory for identical. frictional, nearlt elastic spheres. Physics of Fluids 2002; 14: 1228-1235.

21. Jenkins JT. Dense shearing flows of inelastic disks. Physics of Fluids 2006; 18: 103307.

22. Jenkins JT. Dense shearing flows of inelastic spheres. Granular Matter 2007; 10: 47-52.

23. Poschel T, Buchholtz. Molecular dynamics of arbitrarily shaped granular particles. Journal de Physique I 1995; 5 : 1431-1455.

24. Garzó V, Dufty JW. Hydrodynamics for a granular binary mixture at low density. Annual Review of Fluid Mechanics 1990; 22: 5792.

25. da Cruz F, Emam S, Prochnow M, Roux J, Chevoir F. Rheophysics of dense granular materials: Discrete simulation of plane shear flows. Physical Review E 2005; 72: 021309.

26. Majmudar TS, Behringer RP. Contact force measurements and stress-induced anisotropy in granular materials. Nature 2005; 435: 10791082.

27. Perzyna P. Fundamental problems in viscoplasticity. Advances in Applied Mechanics 1966; 9: 243-377.

28. Muir Wood D. Soil Behaviour and Critical State Soil Mechanics. Cambridge University Press: Cambridge UK, 1990.

29. Kolymbas D. Constitutive Modeling of Granular Materials. Springer: Berlin, 2000.

30. Jop P, Forterre Y, Pouliquen O. Crucial role of sidewalls in granular surface flows: consequences for the rheology. Journal of Fluid Mechanics 2005; 541: 167192.

31. Jop P, Forterre Y, Pouliquen O. A constitutive law for dense granular flows. Nature 2006; 441: 727730.

32. Kamrin K. Nonlinear elasto-plastic model for dense granular flow. International Journal of Plasticity 2010; 26: 167-188.

33. Jiang Y, Liu M. Granular soild hydrodynamics. Granular Matter 2009; 11: 139-156.

34. Johnson P, Jackson R. Frictional-collisional constututive relations for granular materials, with application to plane shearing. Journal of Fluid Mechanics 1987; 176: 67-93. 
35. Johnson P, Jackson R. Frictional-collisional equations of motion for particulate flows and their application to chutes. Journal of Fluid Mechanics 1990; 210: 501-535.

36. Savage SB. Analyses of slow high-concentration flows of granulars. Journal of Fluid Mechanics 1998; 377: 1-26.

37. Louge MY. Model for dense granular flows down bumpy inclines. Physical Review E 2003; 67: 061303.

38. Lee C, Huang C. Model of sheared granular material and application to surface-driven granular flows under gravity. Physics of Fluids 2010; 22: 043307.

39. Berzi D, di Prisco C, Vescovi D. Constitutive relations for steady, dense granular flows. Physical Review E 2011; 84: 031301 .

40. Vescovi D, di Prisco C, Berzi D. From solid to granular gases: the steady state for granular materials. International Journal for Numerical and Analytical Methods in Geomechanics 2013; 37(17): 2937-2951.

41. Roscoe KH, Schofield AN, Wroth CP. On the yielding of soils. Gèotechnique 1958; 8: 22-53.

42. Wroth C. Soil behaviour during shear - existence of critical void ratios. Engineering 1958; 186: 409-413.

43. Schofield A, Wroth C. Critical state soil mechanics. McGraw-Hill: London, U.K., 1968.

44. Been K, Jefferies MG. A state parameter for sands. Gèotechnique 1985; 35(2): 99-112.

45. Houlsby GT. The use of a variable shear modulus in elastic-plastic models for clays. Computers and Geotechnics 1985; 1: 3-13.

46. Loret B. On the choice of elastic parameters for sand. International Journal for Numerical and Analytical Methods in Geomechanics 1985; 9(3): 285-292.

47. Lade PV, Nelson RB. Modelling the elastic behaviour of granular materials. International Journal for Numerical and Analytical Methods in Geomechanics 1987; 11(5): 521-542.

48. Borja RI, Tamagnini C, Amorosi A. Coupling plasticity and energy-conserving elasticity models for clays. Journal of Geotechnical and Geoenvironmental Engineering 1997; 123(1): 948-957.

49. Sulem J, Vardoulakis I, Papamichos E, Oulahna A, Tronvoll J. Elastoplastic modelling of Red Wildmoor sandstone. Mechanics of Cohesive frictional Materials 1999; 4: 215-245.

50. Einav I, Puzrin AM. Pressure-dependent elasticity and energy conservation in elastoplastic models for soils. Journal of Geotechnical and Geoenvironmental Engineering 2004; 130(1): 81-92.

51. Houlsby GT, Amorosi A, Rojas E. Elastic moduli of soils dependent on pressure: a hyperelastic formulation. Gèotechnique 2005; 55(5): 383-392.

52. Vermeer PA, de Borst R. Non-associated plasticity for soils, concrete and rock. Heron, 1984.

53. Simo JC, Huges TJ. Computational Inelasticity, Springer, 1994.

54. Muir Wood D. Geotechnical Modelling. Spon Press: New York, 2004.

55. di Prisco C, Pisano F. An exercise on slope stability and perfect elasto-plasticity. Gèotechnique 2011; 61: 923-934.

56. Chialvo S, Sun J, Sundaresan S. Bridging the rheology of granular flows in three regimes. Physical review E 2012; 85: 021305 .

57. Li XS, Dafalias YF. Dilatancy for cohesionless soils. Gèotechnique 2000; 50(4): 449-460.

58. Jenkins J, Berzi D. Dense inclined flows of inelastic spheres: tests of an extension of kinetic theory. Granular matter 2010; 12(2): 151-158

59. Vescovi D. Granular shear flows: constitutive modeling and numerical simulations. PhD thesis, Politecnico di Milano, Milan, Italy 2014.

60. Silbert L. Jamming of frictional spheres and random loose packing. Soft Matter 2010; 6(13): 2918-2924. 